\title{
Probing Alloy Formation Using Different Excitonic Species: The Particular Case of InGaN
}

\author{
G. Callsen, ${ }^{*}$ R. Butté, and N. Grandjean \\ Institute of Physics, École Polytechnique Fédérale de Lausanne (EPFL), CH-1015 Lausanne, Switzerland
}

(Received 11 October 2018; revised manuscript received 13 February 2019; published 21 August 2019; corrected 21 February 2020)

\begin{abstract}
Since the early 1960s, alloys are commonly grouped into two classes that feature either bound states in the band gap (I) or additional, nondiscrete band states (II). Consequently, one can observe either excitons bound to isoelectronic impurities or the typical band edge emission of a semiconductor that shifts and broadens with rising isoelectronic doping concentration. Microscopic parameters for class I alloys can directly be extracted from photoluminescence (PL) spectra, whereas any conclusions drawn for class II alloys usually remain limited to macroscopic assertions. Nonetheless, we present a spectroscopic study on exciton localization in a mixed-crystal alloy (class II) that allows us to access microscopic alloy parameters. In order to illustrate our approach, we study bulk $\operatorname{In}_{x} \mathrm{Ga}_{1-x} \mathrm{~N}$ epilayers at the onset of alloying $(0 \leq x \leq 2.4 \%)$ in order to understand their robustness to point and structural defects. Through an indepth PL analysis it is demonstrated how different excitonic complexes (free, bound, and complex bound excitons) can serve as a probe to monitor the dilute limit of class II alloys. From an $x$-dependent linewidth analysis we extract the length scales at which excitons become increasingly localized, i.e., their conversion from a free to a bound particle upon alloy formation. Already at $x=2.4 \%$ the exciton diffusion length is reduced to $5.7 \pm 1.3 \mathrm{~nm}$ at a temperature of $12 \mathrm{~K}$; hence, detrimental exciton transfer mechanisms toward nonradiative defects are suppressed. In addition, the associated low-temperature PL data suggest that a single indium atom cannot permanently capture an exciton. The low density of silicon impurities in our samples even allows studying their local indium-enriched environment at the scale of the exciton Bohr radius based on impurity bound excitons. The associated temperature-dependent PL data reveal an alloying dependence for the exciton-phonon coupling. Thus, the formation of the random alloy can not only be monitored by the emission of various excitonic complexes, but also more indirectly via the associated coupling(s) to the phonon bath. Micro-PL spectra even give access to a probing of silicon bound excitons embedded in a particular environment of indium atoms thanks to the emergence of a series of individual and energetically sharp emission lines (full width at half maximum $\approx 300 \mu \mathrm{eV}$ ). Consequently, the present study allows us to extract microscopic properties formerly mostly only accessible for class I alloys.
\end{abstract}

DOI: 10.1103/PhysRevX.9.031030

Subject Areas: Condensed Matter Physics,

Optoelectronics, Semiconductor Physics

\section{INTRODUCTION}

Upon alloying, host atoms in insulators, semiconductors, or metals are replaced by atoms with an equivalent valence electron structure, giving rise to isoelectronic impurities. Despite the matching valency, which does not lead to $n$ - or $p$-type doping in the case of a semiconductor crystal, the total number of electrons is altered, inducing fundamental changes in the electronic band structure and the associated optical signatures of alloying. As already pointed out by Thomas et al. in 1965 [1] and later further conceptualized

\footnotetext{
*gordon.callsen@epfl.ch
}

Published by the American Physical Society under the terms of the Creative Commons Attribution 4.0 International license. Further distribution of this work must maintain attribution to the author(s) and the published article's title, journal citation, and DOI. by Czaja [2] and Dean et al. [3] among others, such isoelectronic impurities can be divided into two classes: For the first class (I), discrete electronic levels are formed in the band gap that can be studied by the related emission of bound excitons in, e.g., GaP:N [1,4], GaP:Bi [5], GaAs:Bi [6], GaAs:N [7,8], ZnTe:O [9], CdS:Te [10], $\mathrm{ZnO}: \mathrm{Hg}[11]$, and GaN: As [12,13]. However, such highly localized bound excitons related to isoelectronic centers strongly differ from the wide range of bound excitons known for shallow impurities in semiconductors and cannot be described by an effective mass approach [14-16]. The second class of isoelectronic centers (II) evokes the formation of mixed-crystal alloys like, e.g., SiGe [17], GaAsP [18,19], InGaAs [20], AlGaAs [21], InGaN [22], AlGaN [23,24], CdSSe [25,26], ZnSeTe [26], and MgZnO [27]. In these cases, no new electronic levels are formed in the band gap, but rather in the bands themselves, a process often described as hybridization [28]. Clearly, such a 
simple classification is not always straightforward [29] and even a continuous, concentration-dependent transition between these two classes of isoelectronic centers has been reported for the unique case of silver halides [30].

The consequences resulting from the existence of these two types of isoelectronic centers in nature for any optical material characterization are pivotal. For class I alloys, the apparent rich optical signature of, e.g., isoelectronic bound excitons [31] allows us to directly extract microscopic material parameters from macroscopic photoluminescence (PL) spectra. Not only the number of isoelectronic impurities per binding center can be extracted $[1,4,6]$, but even the distance in between these impurities or their constellation can be determined [4,32,33]. In contrast, for class II alloys, such spectroscopic analysis is hampered. Commonly, only a continuous shift of the band edge luminescence is observable, which is additionally plagued by a pronounced linewidth broadening [20,21], preventing any detailed information to be extracted from PL spectra. Consequently, in contrast to class I alloys, only a rather indirect analysis of such mixed-crystal alloys is feasible by optical techniques. What further worsens the situation is the fact that isoelectronic impurities related to class II alloys are more abundant in nature in comparison to their type I counterparts [2]. Furthermore, most technologically relevant alloys (e.g., InGaAs, AlGaAs [34], AlInGaP [35], InGaN, InAlN [36], and AlGaN [37]) belong to class II alloys. Among them, especially III-nitride alloys have recently proven their importance for a wide range of applications, rendering a spectroscopic analysis beyond the limits of their classification as mixed-crystal alloys an auspicious task. The apparent difference in the optical signature of these two alloy classes also forms part of the motivation for the employed labeling. Therefore, we aim to denote a ternary alloy in the spirit of a doped binary semiconductor (AB:C) as long as spectrally well-isolated isoelectronic bound excitons dominate the overall band edge emission [4]. Otherwise, we apply the common notation for a ternary semiconductor alloy (ABC).

Over the past decade, III-nitride-based semiconductors have reached a high level of dissemination second only to silicon based on a wide range of applications covering both electronics and optoelectronics [38]. Many aspects of our daily life are already impacted by III-nitride-based lightemitting diodes (LEDs) and laser diodes [39,40], while high-power electronics is also on the rise $[41,42]$. Here, ternary alloys like InGaN and InAlN play a crucial role, as essential properties like, e.g., their emission wavelength can be tuned over a wide range [43] in order to suit the particular application of choice. Such alloys containing indium are often seen as "special alloys" because many devices like LEDs based on InGaN/GaN quantum wells (QWs) perform astonishingly well, with internal quantum efficiencies $\eta_{\text {int }}$ well beyond $80 \%$ [44], even though the threading dislocation density is high with values in the
$10^{8}-10^{9} \mathrm{~cm}^{-2}$ range [45]. Generally, the material proves particularly robust against structural and point defects in comparison to other III-V alloys (e.g., InGaAs, AlGaAs, AlInGaP, etc.) [35], as outlined in the seminal paper of Chichibu et al. [36]. However, the physical origin behind this behavior is yet not well understood, and several possible causes have been discussed in the literature, as detailed in the following.

Early explanations accounting for the particular case of InGaN range from large structural defects like $\mathrm{V}$ pits [46] and indium clusters [47], over indium zigzag chains $[36,48]$, to the particular electronic environment of a single indium atom [28]. All explanations share the common idea that localization of carriers occurs in the alloy, ultimately leading to higher $\eta_{\text {int }}$ values. As soon as carriers are localized, their diffusion to nonradiative centers is suppressed, which in turn enhances $\eta_{\text {int }}$. While larger structural defects can nowadays be excluded for III-nitride materials based on a combination of structural [scanning transmission electron microscopy (STEM), nanoscale secondary ion mass spectroscopy (nano-SIMS), etc.] and optical [cathodoluminescence (CL), microphotoluminescence $(\mu$-PL), etc.] techniques, the material analysis at the few to even subnanometer scale remains challenging. Even though recent technical progress made by atom probe tomography (APT) indicates that $\mathrm{InGaN}$ is a random alloy [49-51], the impact of assemblies of indium atoms like pairs, triplets, and larger sets on the material's optical signature remains unclear.

In this work, we show that even for class II alloys it is possible to access microscopic material parameters from PL and $\mu$-PL spectra. A detailed PL analysis of bulk $\operatorname{In}_{x} \mathrm{Ga}_{1-x} \mathrm{~N}$ epilayers $(0 \leq x \leq 2.4 \%)$ grown on freestanding GaN substrates (dislocation density $\sim 10^{6} \mathrm{~cm}^{-2}$ ) allows us to extract the effective exciton diffusion length $\left(r_{a}\right)$ and its dependence on indium content $(x)$. By analyzing the linewidth broadening of free and bound excitons, one obtains probes that monitor the alloy formation on different length scales determined by $r_{a}$ and the exciton Bohr radius $r_{B}$, hence, in our case encompassing length scales ranging from tens to a few nanometers. With rising $x$ we measure a decrease of $r_{a}$ toward $r_{B}$ using the free exciton $\left(X_{A}\right)$ as a probe for the formation of a random alloy in which the excitonic center-of-mass (c.m.) motion becomes increasingly negligible at cryogenic temperatures. In addition, the exciton-phonon coupling is studied, e.g., for silicon-bound excitons $\left(\mathrm{Si}^{0} X_{A}\right)$, representing an alternative tool to monitor the onset of alloy formation. The spectroscopy of excitons captured at individual indiumrelated centers proves challenging due to an apparent high density of emitters. Hence, we focus on an analysis of $\mu$-PL spectra of individual silicon-bound excitons embedded in particular configurations of indium atoms in their direct vicinity. As a result, we observe a hierarchy of energetically well-defined emission lines $(\mathrm{FWHM} \approx 300 \mu \mathrm{eV})$ that 
originate from excitonic $\mathrm{Si}^{0} X_{A}-\operatorname{In}^{n}$ complexes in the InGaN alloy. Hence, our study opens an original pathway toward a spectroscopic analysis of the microscopic properties of a class II alloy at the nanometer scale by probing distinct configurations of indium atoms at the onset of alloy formation.

The paper is structured as follows. In Sec. II, we first compare the fundamental excitonic properties of a wide range of materials before focusing the motivation to III-V semiconductors represented by indium-doped GaAs and $\mathrm{GaN}$ as representatives of class II alloys. We present and discuss our results in Sec. III. This section is subdivided into five parts labeled Secs. III A-IIIE. In Sec. III A, we show how ensembles of free and bound excitons can serve as probes in the InGaN alloy using PL spectra. Temperature-dependent PL data allow us to analyze the local distribution of indium atoms around particular impurity bound excitons, as demonstrated in Sec. III B. Subsequently, Sec. IIIC introduces the $\mu$-PL traces of individual excitonic complexes bearing on an impurity embedded in a distinct environment of indium atoms. The spectra of such individual bound excitonic complexes are further analyzed in Secs. III D and III E, motivating the existence of spatially direct and indirect excitonic complexes in the InGaN alloy. In a nutshell, Secs. III A-III E describe the following transition. First, ensembles of excitonic complexes are probed in the alloy by PL.
Second, the presented alloy probes become increasingly localized, culminating in the $\mu$-PL observation of individual impurity bound excitonic complexes embedded in a distinct configuration of indium atoms. Section IV finally relates our PL and $\mu$-PL results in a detailed discussion, which motivates future measurements as outlined in the outlook given in Sec. V. Finally, Sec. VI summarizes our findings. Experimental details regarding the spectroscopic and growth techniques can be found in Sec. I of Supplemental Material (SM) [52].

\section{CONTEXT AND MOTIVATION}

In order to obtain a general understanding of the sensitivity of excitons in alloys to crystal defects, Fig. 1(a) introduces the reduction of $r_{B}$ with rising band gap energy $E_{g}$ for a large variety of materials (direct as well as indirect semiconductors and insulators). The higher the ionicity of the crystal-following the transition from IV-IV, over III-V, to II-VI semiconductors- the larger the effective masses of the electrons $\left(m_{e}\right)$ and holes $\left(m_{h}\right)$ that move in the periodic potential of the lattice. In turn, the excitonic effective mass $\mu$ increases accordingly with $E_{g}$ like $1 / \mu=1 / m_{e}+1 / m_{h}$, which is inversely proportional to $r_{B}$ [53]. Such "heavy" excitons with small $r_{B}$ values ultimately lead to the transition from Wannier-Mott-type excitons found in most semiconductors to self-trapped

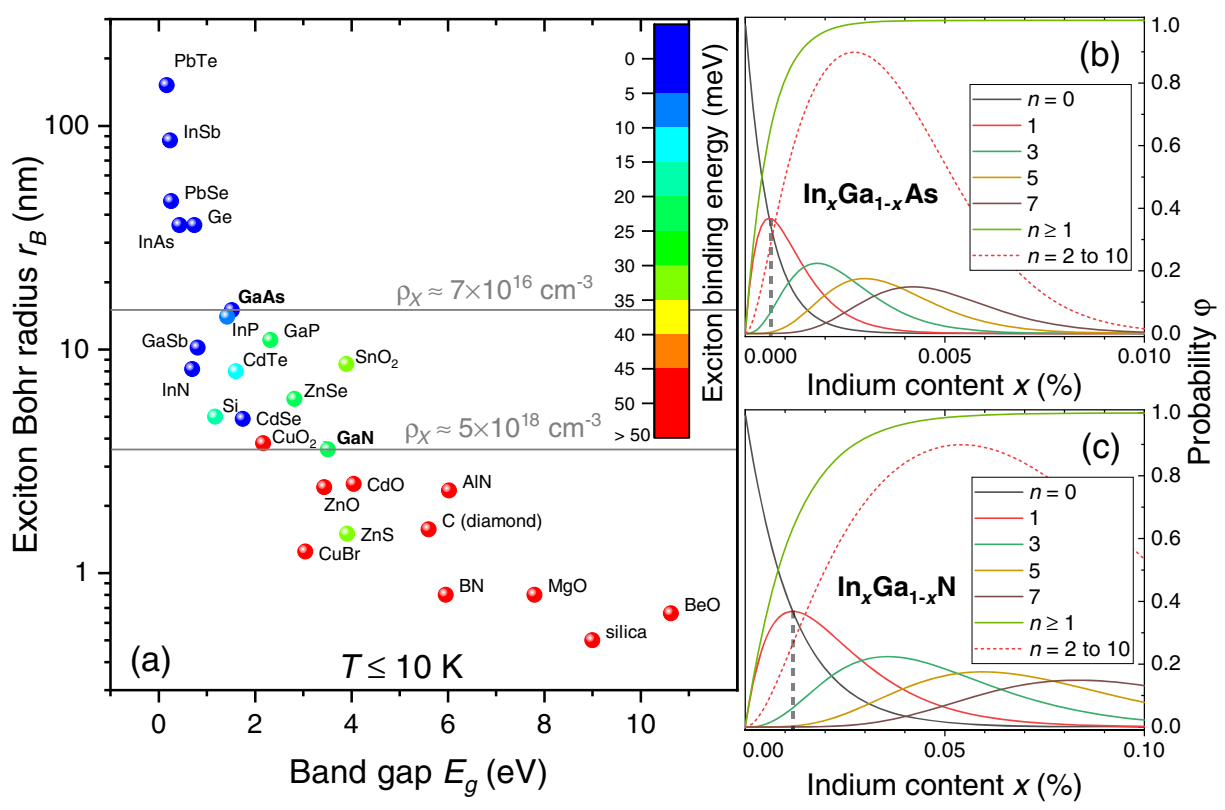

FIG. 1. (a) Hydrogenlike exciton Bohr radius $r_{B}$ (excitonic ground state) for various material systems comprising semiconductors and insulators that exhibit a band gap energy $E_{g}$ between $\approx 0.2$ and $10 \mathrm{eV}$. With diminishing $r_{B}$ and increasing $E_{g}$, the exciton binding energy $E_{\text {bind }}$ rises (color encoded) - a trend which is also valid for all related alloys. The inverse of the exciton volume $V_{X}\left(r_{B}\right)$ motivates the use of a density $\rho_{X}$, which yields a first estimate of the excitons' sensitivity to point and structural defects. To illustrate this matter, we compare $\operatorname{In}_{x} \mathrm{Ga}_{1-x} \mathrm{As}(\mathrm{b})$ and $\operatorname{In}_{x} \mathrm{Ga}_{1-x} \mathrm{~N}$ (c), whose $r_{B}$ values differ by a factor of $\sim 4$. The indium content $x$ required to find a certain number of indium atoms $n$ in $V_{X}$ with the probability $\varphi$ is given by the binomial distribution and differs by one order of magnitude in this comparison. While bound excitons average over $\approx V_{X}$, free excitons monitor the alloy over a larger averaging volume $V_{a}$ due to their eponymous motion. 
excitons frequently observed in silica [54] and halides [55]. As soon as $r_{B}$ is reduced, the exciton binding energy $E_{\text {bind }}$ rises because the Coulomb attraction between the electron and the hole is enhanced. Hence, $E_{\text {bind }}$ is proportional to $\mu$ as encoded in the data point colors of Fig. 1(a), leading to the stability of excitons beyond just cryogenic temperatures for large band gap materials like, e.g., GaN [56] and AlN [57].

Already these basic considerations facilitate some conclusions that should be valid for any alloy of class I or II formed by the materials summarized in Fig. 1(a). In alloys with low $E_{g}$ values, an exciton averages at least over comparably large exciton volumes $V_{X} \propto r_{B}^{3}$. Hence, correspondingly low densities of, e.g., impurities and structural defects $\rho_{X} \propto V_{X}^{-1}$ suffice in order to affect or even trap an exciton if the associated perturbation is sufficiently large. In this sense, $\rho_{X}$ represents a tentative upper limit for the concentration of such centers affecting most excitons. In contrast, high $E_{g}$ materials with lower $V_{X}$ values should be less sensitive to elevated defect concentrations based on this simplistic comparison. In order to illustrate this matter, we highlight the corresponding $\rho_{X}$ values for GaAs and GaN in Fig. 1(a), providing a first sensitivity estimate for excitons in the related alloys. As the $r_{B}$ value of both materials varies by a factor of $\sim 4$, the corresponding approximations of $\rho_{X}$ differ by almost 2 orders of magnitude. However, this comparison is complicated by the occurrence of free, bound (isovalent), and impurity bound (nonisovalent) excitons constituting two- or three-particle complexes. One can observe either free excitons that move and hence monitor the alloy over an averaging volume $V_{a} \geq V_{X}$ leading to lower critical $\rho_{X}$ values or localized, shallow impurity bound excitons sensing the material within $\approx V_{X}$ (see Sec. III A for a detailed analysis). Nevertheless, the lower $r_{B}$ for large $E_{g}$ materials, the less excitons average over a random alloy and the more they just monitor the immediate neighborhood of a distribution of, e.g., (non)isovalent centers in the lattice. This simple picture is further supported by the common neglect of any c.m. motion for excitons in an alloy [58]—a simplification that is addressed in detail in Sec. III A for InGaN.

We further motivate the present study by focusing our previous considerations to InGaAs (e.g., Ref. [20]) and InGaN (this work) as representatives for the cases of relatively high and low $r_{B}$ values in III-V semiconductor alloys of class II, respectively. Based on this choice, the associated $E_{\text {bind }}$ values will remain sufficiently high to ensure a reliable and straightforward PL analysis. The reasoning that further motivates this approach is threefold.

(A) High-quality material is available for $\mathrm{InGaN}$ and InGaAs alloys for studying the onset of alloy formation for class II alloys due to the availability of high-quality substrates for the epitaxial growth of thick ternary layers $(\geq 100 \mathrm{~nm})$ as required for spectroscopy.
(B) It is known that InGaAs- and AlGaAs-based LEDs are rather sensitive to the density of point and structural defects, whereas InGaN proves to be much more robust $[35,36]$ against them.

(C) It was pointed out theoretically that individual indium atoms in GaAs and $\mathrm{GaN}$ do not lead to any bound states in the band gap [28], a matter that would rather require several indium atoms to form more spatially extended binding centers [48].

In other words, both materials are unambiguous representatives of class II alloys with relatively simple spectra that facilitate a detailed tracking of the alloy formation. The bound states otherwise known for class I alloys are mostly associated to single atoms or (extended) pairs and can be observed for a wide range of materials, as summarized in Sec. I. The absence of such optical signatures will allow us to study the very onset of alloy formation in $\mathrm{In}_{x} \mathrm{Ga}_{1-x} \mathrm{~N}$ $(0 \leq x \leq 2.4 \%)$ by monitoring the undisturbed averaging process over the random alloy from the perspective of either free, bound, or impurity (complex) bound excitons.

Generally, the probability $\varphi$ to find $n$ indium atoms in $V_{X}$ for a certain indium content $x$ in a random alloy is given by the binomial distribution describing a typical Bernoulli experiment:

$$
\varphi(n)=\left(\begin{array}{c}
K V_{X} \\
n
\end{array}\right) x^{n}(1-x)^{K V_{X}-n} .
$$

Here, $K V_{X}$ is the cation number in the excitonic volume with $K \approx 0.0438 \AA^{-3}$ for wurtzite $\mathrm{GaN}$ in the indium composition range analyzed in this paper [22,59]. An illustration for the probability $\varphi$ to find, e.g., $0 \leq n \leq 7$ indium atoms in $V_{X}$ is given for InGaN and InGaAs $\left(K \approx 0.0221 \AA^{-3}\right)[21]$ in Figs. 1(b) and 1(c), respectively. The indium content $x$ for which one can find, e.g., one indium atom in $V_{X}$ with $\varphi=0.5$ deviates by more than one order of magnitude, due to the about $4 \times$ larger $r_{B}$ value of GaAs in comparison to GaN. This fact will be crucial for the following PL linewidths analysis of the InGaN alloy, providing insight into the material's particular robustness against point and structural defects [36].

\section{RESULTS}

Figure 2 introduces the low-temperature $(12 \mathrm{~K}) \mathrm{PL}$ spectra of the bulk $\operatorname{In}_{x} \mathrm{Ga}_{1-x} \mathrm{~N}$ sample series at hand with an indium content $x$ ranging from 0 to $2.4 \%$ (see Sec. I in the SM for experimental details [52]). The spectrum of the nonintentionally doped (NID) GaN reference sample shows the common optical traces of the $A$ and $B$ excitons $\left(X_{A}\right.$ and $\left.X_{B}\right)$ along with a dominant neutral donor-bound exciton line $\left(\mathrm{Si}^{0} X_{A}\right)$. Note that based on this assignment we consider the role of exciton polaritons as negligible. This assumption represents an approximation that is motivated by the reported linewidths, which lie above the critical 

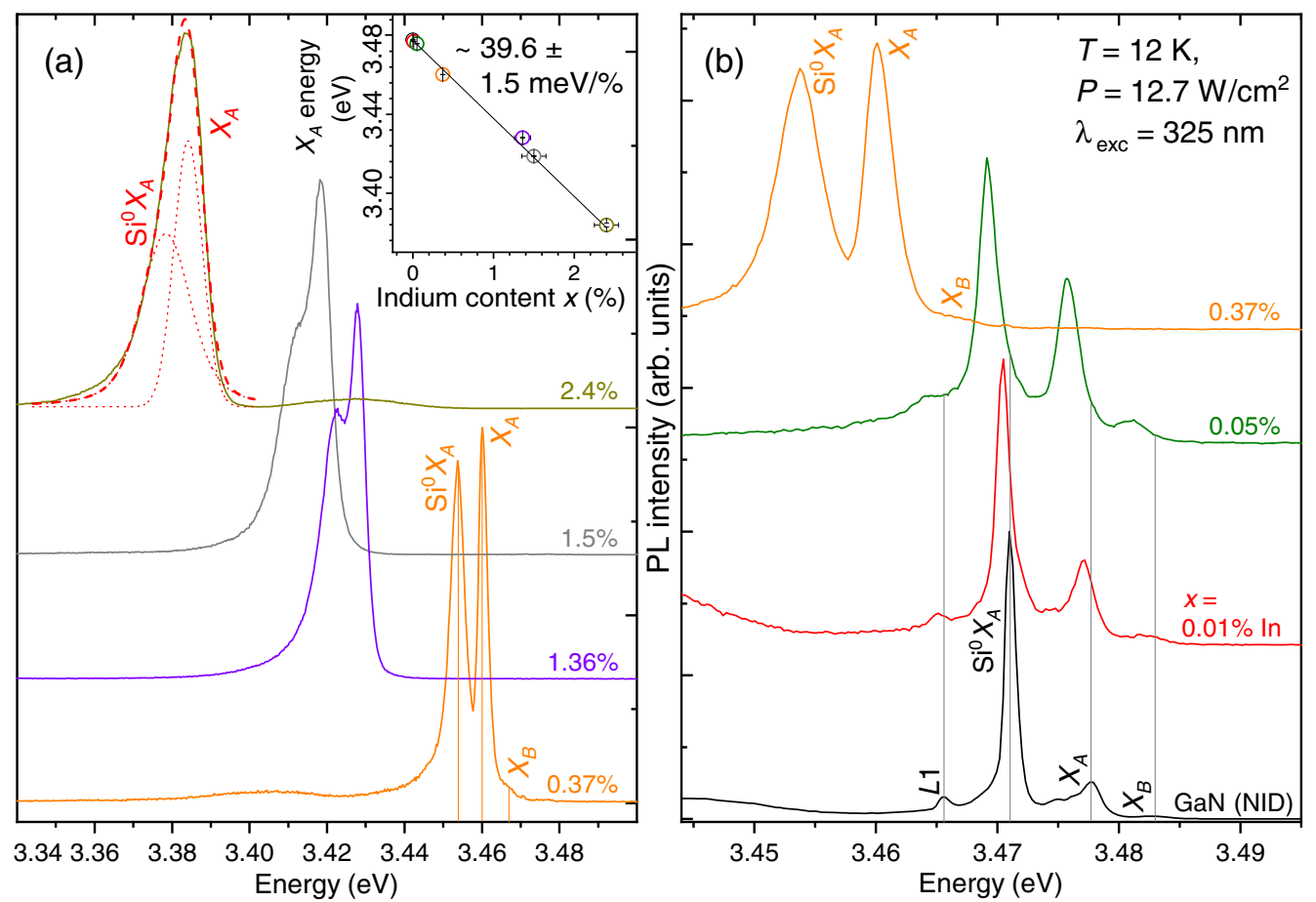

FIG. 2. Overview of PL spectra of the $\operatorname{In}_{x} \mathrm{Ga}_{1-x} \mathrm{~N}$ sample series $(0 \leq x \leq 2.4 \%)$. The energetic shift and the linewidth of the two main emission lines related to the silicon bound exciton $\left(\mathrm{Si}^{0} X_{A}\right)$ and the $A$ exciton $\left(X_{A}\right)$ can be followed up to an indium content of $x=2.4 \%$ in (a) and (b). For the $X_{A}$ transition, a shift rate of $39.6 \pm 1.5 \mathrm{meV} / \%$ is extracted (inset) for the studied composition range at the given temperature of $12 \mathrm{~K}$. Additionally, a faint bound excitonic emission $(L 1)$ and the emission of the $B$ exciton $\left(X_{B}\right)$ can be observed at the very onset of alloying. All relevant transitions are marked by vertical lines. At $x=2.4 \%$ a fitting procedure based on two Voigt profiles exemplarily highlights the main emission peaks. An inversion of the FWHM values related to $\mathrm{Si}^{0} X_{A}$ and $X_{A}$ is directly visible by, e.g., comparing the spectra for $x=0.01 \%$ and $0.37 \%$ in (b).

linewidth for exciton-polariton formation in $\mathrm{GaN}$ [60]. See Sec. II of SM for further details [52]. The splitting in between $X_{A}$ and $\mathrm{Si}^{0} X_{A}$ is known as the localization energy $E_{\mathrm{loc}} \approx 7 \mathrm{meV}$ [61]. Generally, this terminology is not only used for excitons binding to nonisovalent impurities, but also for the localization of excitons in an alloy due to isoelectronic impurities that induce the formation of a potential landscape [36]. In both cases, the emission energy of the excitons exhibits a redshift with respect to $X_{A}$, which will always be described by $E_{\mathrm{loc}}$ in the following. Previous results have shown that the impurity giving rise to the dominant bound excitonic emission is the neutral silicon center $\mathrm{Si}^{0}$, while other typical trace impurities in $\mathrm{GaN}$ like oxygen are negligible in our samples [61]. In addition, toward lower energies one observes the $L 1$ emission line in Fig. 2(b), whose origin is still under debate in literature, ranging from a deep donor-bound exciton, over an ionized donor-bound exciton, to a neutral shallow acceptor-bound exciton [62-65].

Upon increasing indium content, the entire set of emission lines shifts continuously toward lower energies, while the level of detail in the spectra diminishes due to linewidth broadening as commonly observed for a class II alloy. The spectral shift of the $X_{A}$ transition as a function of the change in band gap energy $\delta E_{g}$ with rising $x$ is linear in the given indium content range and amounts to $\delta E_{g} / \delta x=$ $39.6 \pm 1.5 \mathrm{meV} / \%$ at a temperature of $12 \mathrm{~K}$, as shown in the inset of Fig. 2(a). Subsequently, we will utilize this $\delta E_{g} / \delta x$ value, whereas a detailed study on the band gap bowing in InGaN [66] must remain a task for future work. Clear evidence for the emission line $L 1$ is lost at indium contents exceeding $0.05 \%$, before even the spectral separation between $X_{A}$ and $\mathrm{Si}^{0} X_{A}$ vanishes at $x \geq 1.5 \%$. Nevertheless, even at indium contents of $1.5 \%$ and $2.4 \%$ we can still reveal the presence of the $\mathrm{Si}^{0} X_{A}$ centers in the corresponding spectra by a fitting procedure employing two Voigt profiles as exemplified for $x=2.4 \%$ in Fig. 2(a). As a result, the spectral positions of $\mathrm{Si}^{0} X_{A}$ and $X_{A}$ are obtained for $x \geq 0.37 \%$ along with the corresponding FWHM values $\Delta E$. Subsequently, all $\Delta E$ values are summarized in Fig. 3(a), while the spectral positions along with their temperature dependence are depicted in Fig. S3 of SM [52]. In Sec. III B, the temperature dependence of the linewidths is analyzed in the context of Fig. 4. Details regarding the experimental methods, the data analysis, and the error determination can be found in Sec. I of SM [52]. Herein, the fitting routine used to extract all $\Delta E$ values and spectral positions is also exemplified. 

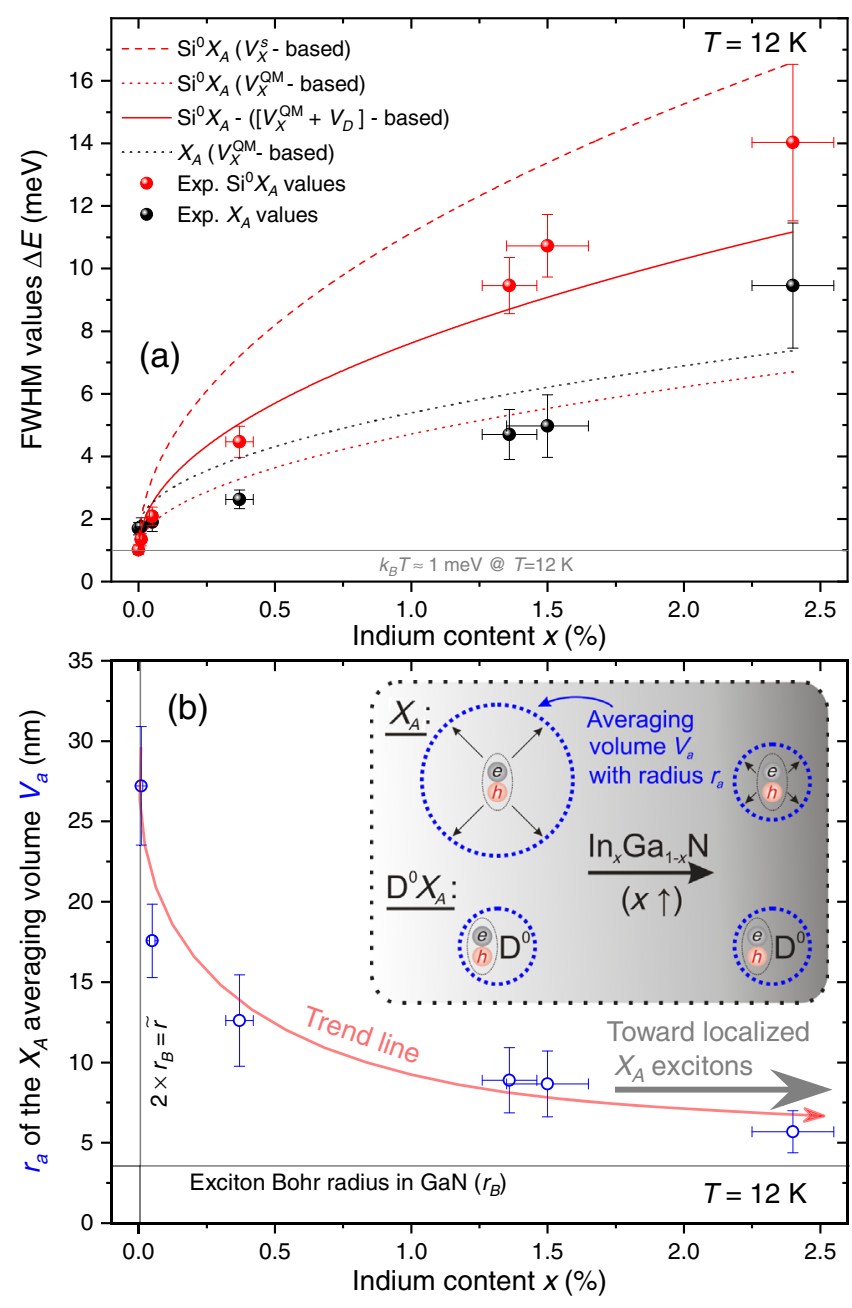

FIG. 3. (a) Experimental FWHM values $\Delta E$ of the $\operatorname{Si}^{0} X_{A}$ (red symbols) and $X_{A}$ (black symbols) transitions versus indium content $x$ extracted from PL spectra. The red lines introduce step by step the modeling of the $\mathrm{Si}^{0} X_{A}$ linewidth depending on the particular excitonic volume $V_{X}$. Here, $V_{X}^{S}$ denotes a spherical and $V_{X}^{\mathrm{QM}}$ the quantum-mechanical exciton volume, while $V_{D}$ is the volume ascribed to the donor electron. The black dotted line shows the corresponding modeling for $X_{A}$ based on $V_{X}^{\mathrm{QM}}$, which deviates from the experimental results $\Delta E$ (black symbols), hence, motivating the concept of a spherical averaging volume $V_{a}\left(r_{a}\right)$. All lines are offset by the experimental $\Delta E$ value for $x=0$. (b) The $r_{a}$ values converge toward the exciton Bohr radius in $\mathrm{GaN}$ because $X_{A}$ gets increasingly localized with rising $x$ in contrast to $\mathrm{Si}^{0} X_{A}$. This basic mechanism is illustrated in the inset. Hence, a random alloy is formed in which the excitonic center-of-mass motion is increasingly suppressed. See the main text for details.

At first glance, the present alloying series of $\mathrm{InGaN}$ seems to match the case of InGaAs as presented by Laurenti et al. [20] for indium contents down to $0.03 \%$. A continuous shift and a continuous broadening of all optical transitions are observed in InGaN down to $x=0.01 \%$, as expected for a class II alloy. Not even at the very onset of the alloying range can one observe additional emission lines related to excitons bound to single isoelectronic indium centers in agreement with the aforementioned theoretical predictions [28]. Note that an indium content of $x=0.01 \%$ yields a probability of $\varphi \approx 0.35$ for a single indium atom to be present in the excitonic volume $V_{X}$, as shown in Fig. 1(c). Hence, the indium content should be sufficiently low to observe the occurrence of additional bound states induced by single indium atoms.

However, a more detailed inspection of PL and $\mu$-PL spectra will result in a manifold of interesting and a priori so-far unforeseen observations for the InGaN alloy, as shown in Secs. III A-III D. The latter mark the transition from an analysis dealing with ensembles of excitons down to individual ones giving access to the microscopic properties of a class II alloy.

\section{A. Probing alloying with free and bound excitons based on macrophotoluminescence}

An in-depth analysis of the emission linewidth of $X_{A}$ and $\mathrm{Si}^{0} X_{A}$ reveals an intriguing feature for $\mathrm{InGaN}$ that is fostered by its large $E_{\text {bind }}$ values and, in comparison to InGaAs, its smaller $r_{B}$ values. At a temperature of $12 \mathrm{~K}$ and $x=0.01 \%$, the $\mathrm{Si}^{0} X_{A}$ transition exhibits a FWHM value of $\Delta E_{\mathrm{Si}^{0} X_{A}}=1.30 \pm 0.06 \mathrm{meV}$, while the FWHM of $X_{A}$ is larger, with $\Delta E_{X_{A}}=1.74 \pm 0.19 \mathrm{meV}$ due to the distribution of free excitons in momentum $(\boldsymbol{k})$ space. Interestingly, with rising indium content this FWHM ratio is reversed as seen, e.g., in the PL spectrum recorded for $x=0.37 \%$, cf. Fig. 2(b). At this indium content we find $\Delta E_{X_{A}}=$ $2.63 \pm 0.04 \mathrm{meV}$, while $\Delta E_{\mathrm{Si}^{0} X_{A}}$ has more than tripled to $4.47 \pm 0.08 \mathrm{meV}$.

The evolution of the experimentally determined $\Delta E$ values for the $X_{A}$ and $\mathrm{Si}^{0} X_{A}$ excitonic complexes is summarized in Fig. 3(a) (black and red symbols, respectively). Both sets of FWHM values show a different evolution as averaging over the alloy occurs for different effective volumes. All $\Delta E$ values are commonly related to the standard deviation $\sigma$ by $\Delta E=2 \sqrt{2 \ln 2} \sigma$. Generally, such $\sigma$ values are composed of an inhomogeneous component due to alloy broadening $\sigma_{E_{g}}(x)$ and a homogeneous, temperature-dependent component $\Gamma(T)$, caused by phonon scattering, leading in first approximation to $\sigma=\sigma_{E_{g}}+\Gamma$ [27,67]. At low temperatures $(12 \mathrm{~K}) \sigma_{E_{g}}$ dominates any phononic effects giving rise to the experimental trends shown in Fig. 3(a) for the directly measured $\Delta E$ values. Additional broadening by $\Gamma(T)$ will subsequently be discussed for $\mathrm{Si}^{0} X_{A}$ in Sec. III B in the context of Fig. 4, while the corresponding analysis for $X_{A}$ is given in Sec. III of SM [52]. Such predominance of inhomogeneous broadening due to alloy disorder at cryogenic temperatures was also observed for other ternary alloys of class II, such as $\mathrm{MgZnO}$ [27], AlGaAs [21], CdZnTe [68], and CdSSe [25].

Based on Eq. (1), the standard deviation of the band gap energy $\sigma_{E_{g}}$ can be expressed by the binomial distribution [21,25]: 


$$
\sigma_{E_{g}}=\delta E_{g} / \delta x \sqrt{\left(\frac{x(1-x)}{K V_{X}}\right)} .
$$

Hence, for a two-particle complex like $X_{A}$ observed at cryogenic temperatures, $\sigma_{E_{g}}$ should equal the standard deviation $\sigma$ determined via $\Delta E$, while $\delta E_{g} / \delta x$ is deduced from Fig. 2(a). As a first approach, $V_{X}$ can be replaced by the standard spherical exciton volume $\left(V_{X}^{s}=4 / 3 \pi r_{B}^{3}\right)$-a method that commonly yields a good agreement with experimental data [27]. However, the quantum mechanical nature of the exciton is completely neglected by this approach, as one assumes a constant occupation probability density for the exciton over $V_{X}$. Instead, the quantummechanical $(\mathrm{QM})$ exciton volume, $V_{X}^{\mathrm{QM}}=10 \pi r_{B}^{3}$, should rather be considered in order to take the wave function of the exciton into account, as outlined in Refs. [25,58].

Figure 3(a) shows that both volumes $\left(V_{X}^{s}\right.$ and $\left.V_{X}^{\mathrm{QM}}\right)$ either lead to a complete overestimation (dashed red line) or underestimation (dotted red line) of the experimentally observed trend for $\mathrm{Si}^{0} X_{A}$, as the effect of the donor electron in this three-particle complex is not yet considered. The $X_{A}$ complex as well as the donor electron sense the alloy formation, leading to energy fluctuations that are normally distributed. Hence, the variance of the $\mathrm{Si}^{0} X_{A}$ peak amounts to $\sigma_{\mathrm{Si}^{0} X_{A}}^{2}=\left[\sigma_{\mathrm{Si}^{0}}\left(V_{D}\right)\right]^{2}+\left[\sigma_{X_{A}}\left(V_{X_{A}}^{\mathrm{QM}}\right)\right]^{2}$. Here, we obtain $V_{D}=10 \pi r_{D}^{3}$ based on Ref. [68] with the donor Bohr radius $r_{D}$ that is derived from the silicon donor binding energy of $28.5 \mathrm{meV}$ [61] calculated within the framework of the hydrogenic model [69]. This consideration of $\mathrm{Si}^{0} X_{A}$ as a three-particle complex leads to a reasonable agreement between experiment and theory [Fig. 3(a), solid red line].

In contrast, for the two-particle complex $X_{A}$ the consideration of $V_{X}^{\mathrm{QM}}$ (dotted black line) still yields an overestimation for most of the experimentally observed $\Delta E_{X_{A}}$ values as the c.m. motion of the exciton would need to be considered at the onset of alloy formation. However, even the theoretical treatment for alloys of Zimmermann given in Ref. [58] deems the c.m. motion of the exciton a task for future work, which has-to the best of our knowledgenever been accomplished. As a direct consequence of the excitons' c.m. motion, the entire set of $X_{A}$ complexes averages over a larger fraction of the alloy. Hence, we suggest to consider an effective spherical averaging volume $V_{a}$ in order to take into account the c.m. motion of the $X_{A}$ complex. This can be understood as an exciton diffusion process (see Sec. IV of SM [52]). Consequently, for the $X_{A}$ complex, Eq. (2) can be solved for $r_{a}$ by replacing $V_{X}$ with $V_{a}=\frac{4}{3} \pi r_{a}^{3}$. As a result, the indium content dependent averaging radius $r_{a}(x)$ is obtained as summarized in Fig. 3(b) (blue circles). Herein, the value of $r_{a}$ is continuously decreasing with rising indium content $x$, as indicated by the light red solid trend line. The photoexcited $X_{A}$ complexes get increasingly localized by indium-related trapping sites until ultimately $r_{a}$ converges toward $r_{B}$. Hence, the free $X_{A}$ exciton transforms into a bound twoparticle complex as shown in Fig. 3(b). In contrast, a $\mathrm{D}^{0} X_{A}$ complex like $\mathrm{Si}^{0} X_{A}$ always remains a bound complex at the onset of alloy formation and should, as a first approximation, maintain an almost constant averaging volume (see the discussion given in Sec. IV of SM [52]). At the onset of alloy formation, we do not expect any strong influence of $x$ on the exciton Bohr radius $r_{B}$. Thus, the $r_{B}$ value shown in Fig. 3(b) corresponds to that of pure $\mathrm{GaN}$, as originally introduced in Fig. 1(a). Singlets, doublets, and with rising $x$ even more extended assemblies of indium atoms [see Fig. 1(c)] should lead to such increasing localization of $X_{A}$ with rising $x$. The average distance in between indium atoms is given by $\tilde{r}(x)=(K x)^{-1 / 3}$. Thus, if a single indium atom were capable of permanently trapping the $X_{A}$ particle at the temperature of interest, then $r_{a}$ should reach $r_{B}$ as soon as $\tilde{r}(x)$ approaches $2 r_{B}$. However, this is clearly not the case, as highlighted by the corresponding vertical gray solid line in Fig. 3(b). Thus, larger assemblies of indium atoms in $V_{a}$ are required to observe the localization of $X_{A}$. In this respect, the $r_{a}(x)$ values represent a measure of the $X_{A}$ diffusion length, as further analyzed in Sec. IV of SM [52]. Let us note that according to Bellaiche et al. [28], the hole as a building block of the $X_{A}$ complex can get localized even in the case of a single indium atom. However, the corresponding energy level is still hybridized with the valence band states of $\mathrm{GaN}$, meaning that no additional states appear in the band gap at the onset of alloy formation, in agreement with our observations from Fig. 2 (no new emission lines appear with rising $x$ ) and our expectations for a class II alloy. This particular case is fundamentally different from that of excitons bound to isoelectronic centers (cf. examples given in Sec. II) mostly situated on anionic lattice sites [31], whose strong localization leads to the appearance of new energy levels in the band gap. Finally, about $2 \%-3 \%$ of indium seems to be required to form an InGaN alloy for which the neglect of the excitons' c.m. motion [58] is justified at cryogenic temperatures. However, with increasing $x$, additional broadening mechanisms beyond a pure alloying-induced broadening also start to affect $\Delta E_{X_{A}}$. As discussed by Butté et al. [22], such structural broadening mechanisms could increasingly affect the measured $\Delta E_{X_{A}}$ values with rising $x$.

This transitional regime of increasing excitonic localization at $12 \mathrm{~K}$ by indium-related assemblies is challenging from a theoretical point of view, as exciton diffusion needs to be considered. The common assumption of a phononassisted hopping or tunneling process of the excitons between localization centers forms the basis for modeling exciton diffusion [70,71]. Section IV of the SM [52] summarizes the particular challenges for InGaN and connects our experimental findings with this modeling approach, which was originally developed for the aforementioned, strongly localized excitons bound to isoelectronic centers. As a 
result, a first comparison of our results for the $\mathrm{InGaN}$ alloy with the findings of, e.g., Müller et al. for $\mathrm{MgZnO}$ and CdSSe, is obtained [72]. Even though information about exciton diffusion can be deduced from a PL line shape analysis [73] of the alloy emission, e.g., via time-resolved PL experiments [72], such studies lie beyond the scope of the present work.

Furthermore, we plausibly assume that our findings from Fig. 3 related to the localization of $X_{A}$ with rising $x$ are connected to the dilute indium assemblies (e.g., indium zigzag chains [48]) described by Chichibu et al. [36]. However, their precise substructure at the atomic scale still remains difficult to access. In this regard, Secs. III C and III D describe a pathway toward an analysis of the particular configuration of indium atoms by spectroscopic means at the onset of alloy formation. In the following, we always refer to such dilute indium assemblies, because larger, nondilute indium aggregates should lead to the formation of electronic states in the band gap [48] [i.e., quantum-dot- (QD) like states].

We suggest that the apparent localization of $X_{A}$ complexes with rising $x$ enhances the robustness of the InGaN alloy against point and structural defects in contrast to other III-V alloys, as outlined by Chichibu et al. in Ref. [74]. Such a picture also accounts for the intensity increase of $X_{A}$ relative to $\mathrm{Si}^{0} X_{A}$, as shown in Fig. 2. As exciton diffusion is inhibited with rising $x$, the probability to reach nonradiative exciton trapping sites is reduced. In this respect, we wish also to note that the overall intensity of the band edge luminescence shown in Fig. 2 continuously increases with rising indium content at the onset of alloy formation (not shown) as frequently reported in the literature [75-78]. Naturally, with rising $x$ the average trapping potential becomes deeper as larger indium assemblies are formed that ultimately even govern the $\boldsymbol{k}$ distribution of $X_{A}$ at a temperature of $300 \mathrm{~K}$ with localization energies already in excess of $k_{B} T$ at $x \approx 3 \%$ [22]. In addition, larger indium assemblies can not only lead to exciton localization but also to confinement, which commonly boosts excitonic decay rates. Such an inhibited exciton diffusion should also be relevant for studying the impact of threading dislocations, which could directly be analyzed by means of, e.g., CL in bulk $\operatorname{In}_{x} \mathrm{Ga}_{1-x} \mathrm{~N}$ epilayers with rising $x$. Interestingly, for a $2-\mu \mathrm{m}$-thick $\mathrm{GaN}$ layer grown on freestanding GaN substrates, Liu et al. determined an exciton diffusion length of $\approx 24 \mathrm{~nm}$ [79] based on time-resolved CL measurements. Their finding matches our result of $r_{a}=27 \pm 3 \mathrm{~nm}$ from Fig. 3(b) for our most dilute sample with $x=0.01 \%$, which approaches the case of pure GaN. Hence, Fig. 3 points toward the determination of exciton diffusion length in an alloy based on rather simple macro-PL measurements. The resulting data seem of more general nature as the trapping of excitons is monitored over a large excitation area (excitation spot diameter of $\approx 100 \mu \mathrm{m}$ ) and does not depend on the specific strain-driven impurity distribution around a structural defect [80]. Obviously, such increasing exciton localization with rising $x$ is less beneficial for alloys like $\mathrm{In}_{x} \mathrm{Ga}_{1-x}$ As as already much larger $r_{B}$ values prevail in the related binary compounds as introduced in Fig. 1(a).

\section{B. Analysis of the local indium distribution around impurity bound excitons}

The transition from a free to a bound excitonic twoparticle complex summarized in Fig. 3(b) represents a spectroscopic probe, whose sensitivity to the local environment in the alloy increases with rising indium content as $r_{a}$ converges toward $r_{B}$. In contrast, an impurity bound three-particle complex always remains localized in the dilute alloy limit, giving rise to a permanent local alloy probe, as illustrated in the inset of Fig. 3(b). The limit of this assumption is further discussed in Sec. IV of SM [52].

Nevertheless, in the following we show that the impurity bound exciton complex $\mathrm{Si}^{0} X_{A}$ also represents an extremely versatile tool for studying the formation of dilute indium assemblies. While the temperature-dependent linewidth of $X_{A}$ does not show any clear indium content-dependent trend (see Fig. S4 in the SM [52]), the broadening of $\mathrm{Si}^{0} X_{A}$ upon rising temperature increases with $x$, as shown in Fig. 4(a) - an interesting finding in light of previous alloy studies focusing on $\mathrm{MgZnO}$ [27]. In addition to the data shown in Fig. 4, Secs. II and III of SM provide an overview about the underlying temperature-dependent PL spectra and the corresponding analysis of peak positions and linewidths [52].

Generally, the phonon-induced linewidth broadening $\Gamma(T)$ is given by $[56,67,81]$

$$
\Gamma(T)=\gamma_{\mathrm{ac}} T+\sum_{i=1}^{2} \frac{\gamma_{\mathrm{opt}}^{i}}{\exp \left(E_{\mathrm{opt}}^{i} / k_{B} T\right)-1},
$$

with the acoustical $\left(\gamma_{\text {ac }}\right)$ and the optical $\left(\gamma_{\text {opt }}^{i}\right)$ phonon coupling constants as well as the corresponding effective optical phonon energies $\left(E_{\text {opt }}^{i}\right)$ each numbered by $i$. Equation (3) originates from linear response theory, which allows deriving the relevant exciton-phonon scattering rates as described, e.g., in Refs. [82,83]. While the second term of Eq. (3) sums up the impact of different optical phonons, the first term already comprises the interaction of excitons with acoustic phonons based on the deformation potential and the piezoelectric couplings [83,84]. Viswanath et al. [56] provided a detailed literature overview for the major theoretical contributions that form the basis for Eq. (3), which has commonly been applied to describe, e.g., the broadening of excitonic emission lines for a number of binary semiconductors [56,84-86] and also ternary semiconductor alloy systems $[27,87]$.

The best fit to the evolution of $\Delta E(T)$ shown in Fig. 4(a) for $\mathrm{Si}^{0} X_{A}$ at $x=0$ (solid black line) is obtained for $\gamma_{\mathrm{ac}}=$ $4 \pm 1 \mu \mathrm{eV} / \mathrm{K}$ and only one set of $\gamma_{\mathrm{opt}}^{1}, E_{\mathrm{opt}}^{1}$ values $(i=1)$. Hence, in total three fitting parameters are applied for 

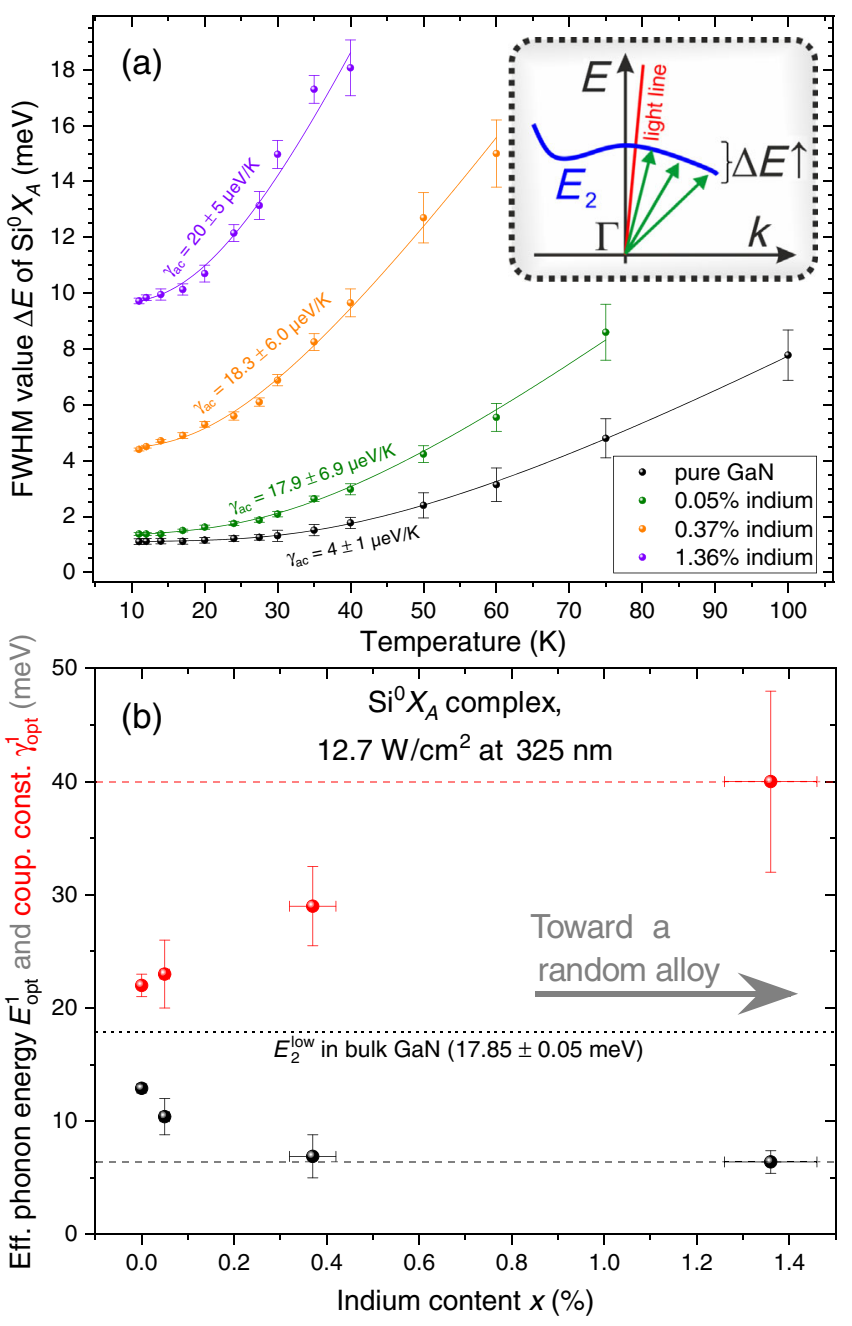

FIG. 4. (a) Temperature dependence of the linewidth broadening for the $\mathrm{Si}^{0} X_{A}$ complex extracted from PL spectra. Solid lines show fits to the data based on Eq. (3). The value of the fitting parameter $\gamma_{\mathrm{ac}}$ is reported, whereas the particular scaling behavior of the fitting parameters $E_{\mathrm{opt}}^{1}$ and $\gamma_{\mathrm{opt}}^{1}$ is shown in (b). Here, the values for $E_{\mathrm{opt}}^{1}$ and $\gamma_{\mathrm{opt}}^{1}$ seem to converge with rising $x$ (dashed lines). The temperature dependence of the linewidth broadening for $\mathrm{Si}^{0} X_{A}$ is strongly affected by the indium content in the alloy $x$, in contrast to the case of $X_{A}$ (cf. Fig. S4 in the SM [52]). An optical phonon population with an effective energy $E_{\mathrm{opt}}^{1}$ (weighted by the corresponding density of states) is responsible for the particular broadening of $\mathrm{Si}^{0} X_{A}$. The maximum energy of phonons within this phonon population corresponds to the energy of the $E_{2}^{\text {low }}$ phonon in $\mathrm{GaN}(17.85 \pm 0.05 \mathrm{meV})$. The inset in (a) sketches the $E_{2}$ phonon dispersion relation in the first Brillouin zone near the $\Gamma$ point, cf. Sec. IIIB.

each fit function shown in Fig. 4(a). Additional fitting parameters would only need to be considered for higher temperatures and the interconnected activation of, e.g., longitudinal-optical (LO) phonons as demonstrated for $X_{A}$ in Sec. III of SM [52] for temperatures up to $300 \mathrm{~K}$. The derived value for $E_{\mathrm{opt}}^{1}=12.9 \pm 0.4 \mathrm{meV}$ for the case of pure $\mathrm{GaN}$ [solid black line in Fig. 4(a)] is smaller than the energy of the $E_{2}^{\text {low }}$ phonon mode in GaN of $17.85 \pm$ $0.05 \mathrm{meV}$ [88]. This difference can mainly be explained by the curvature of the corresponding phonon dispersion relation $E(k)$ around $k_{0} \approx 0$ with $\partial^{2} E /\left.\partial k^{2}\right|_{k=k_{0}}<0$, as illustrated in the inset of Fig. 4(a) [89]. The exciton-phonon coupling related to $\mathrm{Si}^{0} X_{A}$ probes an extended phonon energy interval due to the localization of the bound exciton in real space, providing access to a larger fraction of the first Brillouin zone near the $\Gamma$ point. Hence, the temperature-dependent PL data yield an effective phonon energy $E_{\mathrm{opt}}^{1}$ that is weighted by the corresponding onephonon density of states [90-92]. Clearly, the possibility to observe this phenomenon is enabled by the comparably small $r_{B}$ value of GaN as introduced in Fig. 1(a), which leads to a pronounced extension of the exciton in $\boldsymbol{k}$ space. A more precise determination of $r_{B}$ would even yield lower values if polaronic effects [93] and the binding potential of the silicon impurity [61] were taken into account. However, a quantification of the corresponding weightings for the $\mathrm{Si}^{0} X_{A}$ complex remains a task for future work.

Interestingly, the decrease of $E_{\mathrm{opt}}^{1}$ upon alloying is more pronounced than the aforementioned phenomenon induced by exciton localization in pure GaN. For $\mathrm{Si}^{0} X_{A}$ the effective phonon energy $E_{\text {opt }}^{1}$ halves as depicted in Fig. 4(b) by gradually decreasing from $12.9 \pm 0.4$ to $6.4 \pm 1.0 \mathrm{meV}$ over the composition range $0 \leq x \leq 1.36 \%$. Here, Fig. 4(b) also shows that the corresponding coupling constant $\gamma_{\mathrm{opt}}^{1}$ rises continuously upon the decline of $E_{\mathrm{opt}}^{1}$. The observation for $E_{\mathrm{opt}}^{1}$ is of particular interest as the corresponding $E_{\text {loc }}$ value of $\mathrm{Si}^{0} X_{A}$ seems almost unaffected at the onset of alloy formation, as shown in Fig. 2 and Fig. S2 of SM [52]. A careful inspection of the spectra yields a reduction in $E_{\text {loc }} \lesssim 1 \mathrm{meV}$ for the alloying interval of interest. Therefore, the observed reduction in $E_{\text {opt }}^{1}$ is not predominantly induced by a rise in exciton localization, which would otherwise scale with the overall complex binding energy $E_{\text {bind }}$ and consequently $E_{\text {loc }}$ via Haynes's rule [94] for shallow donor-bound excitons in $\mathrm{GaN}$ [95]. In addition, the corresponding $\gamma_{\mathrm{ac}}$ fitting parameter denoted in Fig. 4(a) continuously rises with $x$ and approaches values equal to the ones observed for $X_{A}$ within the given error bars (see Fig. S4 and the corresponding Sec. III in SM [52]).

Generally, the observed trend for $E_{\mathrm{opt}}^{1}$ likely originates from an alloying-induced local variation of the $\mathrm{Si}^{0}$ environment. Callsen et al. have found for single excitons trapped in GaN/AlN QDs that, e.g., the exciton-LOphonon interaction averages over a volume with a radius on the order of $r_{B}$ [96]. Hence, alloying in thick InGaN epilayers can not only directly be monitored by, e.g., emission energy shifts (Fig. 2) and linewidths broadenings (Fig. 3), but also more indirectly by the exciton-phonon coupling as evidenced by the data obtained from Fig. 4. As $\Gamma(T)$ for $X_{A}$ does not show any pronounced indium content 
dependence (see Fig. S4 in the SM [52]), we suggest that the environment of $\mathrm{Si}^{0}$ centers is richer in indium atoms, a feature only noticeable at the very onset of alloy formation. Thus, we can reasonably expect that the phonon energies and the corresponding coupling constants (e.g., $E_{\text {opt }}^{1}$ and $\gamma_{\text {opt }}^{1}$ ) shall rapidly converge with rising $x$. Indeed, any local variation in the indium content caused by a particular distribution of $\mathrm{Si}^{0}$ atoms will get increasingly masked by random alloy fluctuations with rising $x$. As a signature of this masking, $E_{\text {opt }}^{1}(x)$ converges toward $\approx 6 \mathrm{meV}$, as shown in Fig. 4(b), while the rise in the optical phonon coupling constant $\gamma_{\mathrm{opt}}^{1}$ also seems to saturate with increasing $x$ values.

As a possible reason for the local indium enrichment in the vicinity of $\mathrm{Si}^{0}$ centers, we suggest tensile strain that is commonly introduced upon silicon doping of GaN [97]. Hence, it is energetically more favorable for a rather large atom like indium to incorporate close to a silicon atom (distance on the order of $r_{B}$ ), in order to approach the lattice strain equilibrium at the onset of the alloy formation. Bezyazychnaya et al. have theoretically predicted the impact of point defects (vacancies) in $\mathrm{InGaN}_{\text {and }}$ InGaAs on the indium distribution in these alloys [98]. Here, we experimentally find-to a certain degree- a similar situation for the $\mathrm{Si}^{0}$ impurity. Future theoretical work is needed to validate this picture of a point defect embedded in a dilute assembly of indium atoms that extends on the order of $r_{B}$. This image differs from the concept of direct complex formation [99] that often just considers nearest- or second-nearest-neighbor sites on the cationic sublattice.

Clearly, the total decrease in the effective phonon energy $E_{\text {opt }}^{1}$ cannot exclusively be explained by a local rise in indium content around the $\mathrm{Si}^{0}$ impurity as even pure $\mathrm{InN}$ still exhibits an $E_{2}^{\text {low }}$ phonon energy of around $10.9 \pm$ $0.1 \mathrm{meV}$ [89]. In addition, nondilute indium assemblies matching the size of $V_{X}$ could have been detected by APT in state-of-the-art InGaN/GaN QW samples $[49,50]$ that commonly comprise silicon concentrations $\approx 1 \times$ $10^{16} \mathrm{~cm}^{-3}$ (cf. Sec. I of SM [52]). Thus, we suggest that the reduction in the $E_{\mathrm{opt}}^{1}$ values shown in Fig. 4(b) is related to phonon localization [100] induced by the isoelectronic centers surrounding the $\mathrm{Si}^{0}$ center within $\sim r_{B}$. As a direct consequence, the translational invariance of the crystal is affected, which in turn partly localizes the phonon modes around the impurity. At this point, the common concept of phonon dispersion relations as indicated in the inset of Fig. 4(a) becomes obsolete, as more and more localized phonon modes are formed with rising $x$. These modes presumably originate from the bulk $E_{2}^{\text {low }}$ phonon mode of $\mathrm{GaN}$, as suggested by the gradual decrease of $E_{\mathrm{opt}}^{1}$ with rising $x$, cf. Fig. 4(b). To a certain extent, this picture is similar to the common concept of phonon confinement in nanostructures $[96,101,102]$ that leads, e.g., to a backfolding of phonon branches. Consequently, a gradual increase in the localization of the phonon modes should indeed lead to the observed decrease in the phonon energy $\left(E_{\mathrm{opt}}^{1}\right)$ and to an enhanced exciton-phonon coupling strength $\left(\gamma_{\mathrm{opt}}^{1}\right)$, which scales with the overlap between the exciton wave function and the particular phonon mode of interest [96].

Even more direct experimental evidence for the interaction between localized phonon modes and the $\mathrm{Si}^{0} X_{A}$ is given in the next section. In any case, those results highlight the need for future theoretical work on the localization of phonons due to isoelectronic centers situated around an impurity like $\mathrm{Si}^{0}$. The observations reported in Fig. 4 also motivate additional experimental work in order to directly access such localized phonons by Raman spectroscopy, as outlined in Sec. V.

\section{Individual bound excitonic complexes analyzed by microphotoluminescence}

Micro-PL measurements with an excitation spot diameter $\approx 1 \mu \mathrm{m}$ can provide more detailed information regarding the particular configurations of indium atoms close to a $\mathrm{Si}^{0}$ donor at the onset of alloy formation. Even on completely unprocessed samples one can already resolve individual emission lines around the $\mathrm{Si}^{0} X_{A}$ transition for $x=0.37 \%$ and partially for $1.36 \%$, as shown in Fig. 5(a), while the $X_{A}$ emission remains a rather unstructured emission band due to the given probe volume. This observation is of high importance as the subsequently described processing of metal apertures on the sample could lead to unwanted exciton localization induced by any damage to the sample, as discussed in Sec. V of SM [52].

We further reduced the size of the probe volume by processing apertures into an aluminum film with diameters down to $200 \mathrm{~nm}$ on the sample with $x=0.37 \%$. As a result, a set of spectrally well-separated emission lines can be resolved around $\mathrm{Si}^{0} X_{A}$ along with particular phonon sidebands, as shown in Fig. 5(b). These phonon sidebands appear on the low-energy side of the set of sharp emission lines as an equally spaced $(\approx 18 \mathrm{meV})$ double step. The present energetic spacing already suggests a significant contribution of phonons that originate from the bulk $E_{2}^{\text {low }}$ host mode. Interestingly, the optical signature of the exciton-phonon coupling does not appear as a commonly observed [95] isolated peak [see the first LO replica of $X_{A}$ in Fig. 5(b)], but as a step due to the energetically broad range of contributing phonons. This observation is in line with the results of Fig. 4, which yielded the prediction of a non-negligible contribution of localized phonons with lower energies due to the presence of isoelectronic centers in the immediate $\mathrm{Si}^{0}$ environment. A careful inspection of the spectrum from Fig. 5(b) for $x=0.37 \%$ yields even more details regarding the contribution of localized phonons to the exciton-phonon coupling. About $7 \mathrm{meV}$ 


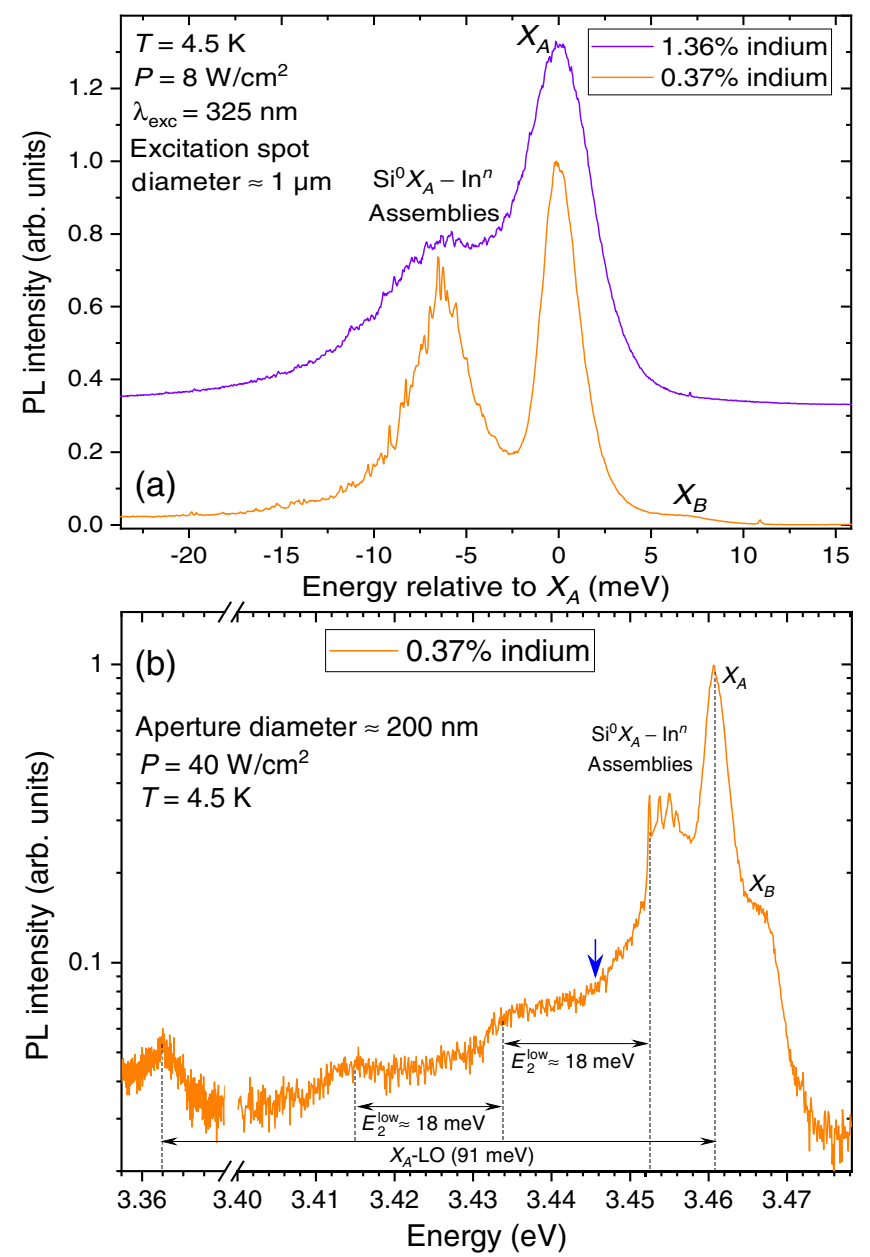

FIG. 5. (a) $\mu$-PL spectra of two samples containing $0.37 \%$ and $1.36 \%$ of indium, respectively, show a structured spectrum in the energy range of $\mathrm{Si}^{0} X_{A}$, while the emission band related to $X_{A}$ remains unstructured (spectra are shown on a relative energy scale for better comparison). Both spectra are typical for the entire bare surface area of our samples. The density of sharp emission lines noticeable around $\mathrm{Si}^{0} X_{A}$ increases with rising $x$. (b) At an indium content of $x=0.37 \%$ and a temperature of $4.5 \mathrm{~K}$, the $X_{A}$ and $X_{B}$ transitions still appear as unstructured bands even if measured under a metal aperture with a diameter of $200 \mathrm{~nm}$ by means of $\mu$-PL. In contrast, the $\mathrm{Si}^{0} X_{A}$ emission band falls apart into sharp emission lines with FWHM values down to $\approx 300 \mu \mathrm{eV}$. At the given low excitation density $\left(\sim 40 \mathrm{~W} / \mathrm{cm}^{2}\right)$, several phonon sidebands are noticeable $\left[E_{2}^{\text {low }}\right.$-related and longitudinal-optical (LO) phonon replica]. Here, the blue arrow indicates a particular, effective phonon energy $E_{\mathrm{opt}}^{1}=6.9 \mathrm{meV}$ as extracted in Fig. 4(b) for $x=0.37 \%$.

below the first step related to the zero phonon lines [marked by a blue arrow in Fig. 5(b)] one even observes an additional, gradual increase in intensity. This spacing seems to correspond to the effective phonon energy $E_{\text {opt }}^{1}=6.9 \mathrm{meV}$ derived in Fig. 4(a) for $x=0.37 \%$. Hence, the $\mu$-PL spectrum shown in Fig. 5(b) seems to represent a coarse probe of the phonon density of states interlinked with the exciton-phonon coupling strength associated to $\mathrm{Si}^{0} X_{A}$, which directly relates to the corresponding modeling of homogeneous linewidth broadening $\Gamma(T)$ introduced in Fig. 4(a).

The sharp emission lines in Fig. 5(b) present the most direct evidence for an alloy-induced perturbation of the immediate $\mathrm{Si}^{0}$ environment at $x=0.37 \%$, which has led to the aforementioned localization of phonons. Emission lines with a linewidth of just $320 \pm 30 \mu \mathrm{eV}$ can be resolved at the given low excitation power density $\left(\sim 40 \mathrm{~W} / \mathrm{cm}^{2}\right)$, opening the perspective for a spectroscopic study of the $\mathrm{Si}^{0}$ environment at the nanometer scale based on the emission of such dilute $\mathrm{Si}^{0} X_{A}-\operatorname{In}^{n}$ assemblies with $n$ indium atoms. In contrast to the emission around $\mathrm{Si}^{0} X_{A}$, the $X_{A}$ emission remains an unstructured band in our $\mu$-PL spectra. Two effects contribute to this observation: the different densities of the binding centers and the sufficiently low inhomogeneous broadening of the $\mathrm{Si}^{0} X_{A}-\mathrm{In}^{n}$ related bound excitonic emission lines. First, the number of $X_{A}$ complexes that attach to a certain indium atom configuration $\operatorname{In}^{n}$ is comparably larger than the corresponding number of $\mathrm{Si}^{0} X_{A}-\mathrm{In}^{n}$ centers that occur in the $\mu$-PL probe volume, cf. Sec. I of SM [52]. Second, the inhomogeneous linewidth is, with $320 \pm 30 \mu \mathrm{eV}$, sufficiently low to observe individual $\mathrm{Si}^{0} X_{A}-\operatorname{In}^{n}$ related emitters, cf. Figs. 5(b) and 6. This matter evidences a high sample quality, as record linewidths for impurity bound excitons in pure $\mathrm{GaN}$ are also just of around $100 \mu \mathrm{eV}$ [103]. Hence, only the $\mathrm{Si}^{0} X_{A}-\operatorname{In}^{n}$ emission band falls apart into individual emission lines in the $\mu$-PL spectra of Fig. 5, while a similar observation for $X_{A}$ would require an even lower excitation density and, in the best case, a smaller probe volume (see Sec. V). For instance, the impact of individual indium atoms on the emission of $\mathrm{Si}^{0} X_{A}$ could possibly be resolved for smaller indium contents (e.g., $x=0.01 \%$ ). However, the temperatureinduced emission line shifts shown in Fig. S2 and summarized in Fig. S3 (comparison between $x=0,0.37 \%$, and $1.36 \%$ ) suggest corresponding $E_{\mathrm{loc}}$ values $\lesssim 0.5 \mathrm{meV}$ [52]. Hence, the emission energy difference between, e.g., $\mathrm{Si}^{0} X_{A}$ and $\mathrm{Si}^{0} X_{A}-\operatorname{In}^{n}$ centers at $x=0.01 \%$ cannot straightforwardly be resolved based on the present emission linewidths of our samples $(\approx 300 \mu \mathrm{eV})$. So far, the impact of individual indium atoms can only be monitored by the localization onset of $X_{A}$, as demonstrated in Figs. 3(a) and 3(b), and the altered exciton-phonon coupling already noticeable at $x=0.05 \%$, cf. Fig. 4(a).

\section{Statistical analysis of individual bound excitonic complexes}

The observation of sharp emission lines in Fig. 5 caused by dilute $\mathrm{Si}^{0} X_{A}-\operatorname{In}^{n}$ assemblies directly evokes the need for a statistical analysis of the underlying emitters. Figure 6(a) shows selected $\mu$-PL spectra recorded for four different metal apertures labeled $A 1-A 4$. Here, the two spectra for aperture $A 4$ (measured using the first and second 


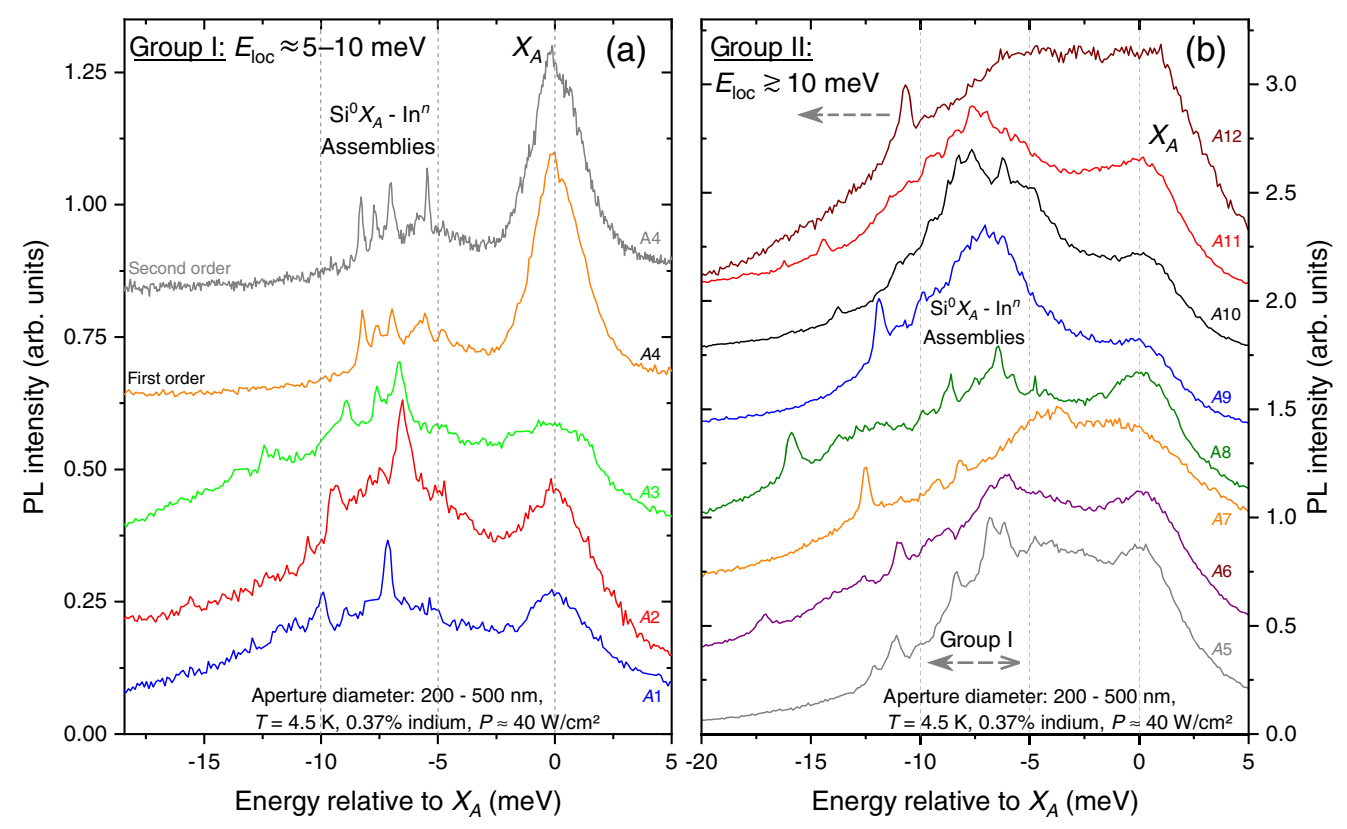

FIG. 6. The sharp emission lines introduced in Fig. 5 appear due to the formation of dilute $\mathrm{Si}^{0} X_{A}-\mathrm{In}^{n}$ assemblies and can be divided into two major groups, I and II, based on $\mu$-PL spectra. (a) Emission lines of group I exhibit localization energies $E_{\text {loc }}$ of $\approx 5-10 \mathrm{meV}$ and show small energetic shifts upon increasing excitation power density. (b) In contrast, emission lines of group II typically show $E_{\text {loc }}$ values in excess of $10 \mathrm{meV}$ and exhibit pronounced spectral shifts for any variation in the excitation power density. This difference in the peak shift rates for group I and II emission lines is highlighted in Fig. 7. Generally, the emission lines of group I appear more frequently than their more deeply localized group II counterparts. $A 1-A 12$ denote the particular submicron metal apertures selected from more than 50 .

order of the optical grating) provide a detailed view of the $\mu$-PL spectrum shown in Fig. 5(b). All spectra displayed in Fig. 6(a) show a common optical signature with sharp emission lines related to dilute $\mathrm{Si}^{0} X_{A}-\operatorname{In}^{n}$ assemblies in addition to the rather unstructured emission band related to $X_{A}$. The localization energy $E_{\text {loc }}$ (spacing between $X_{A}$ and $\mathrm{Si}^{0} X_{A}-\mathrm{In}^{n}$ assemblies) commonly ranges between 5 and $10 \mathrm{meV}$ for these emitters that we assign to group I. Figure 6(b) introduces eight additional $\mu$-PL spectra $(A 5-A 12)$ that also show emission lines with $E_{\mathrm{loc}} \gtrsim$ $10 \mathrm{meV}$ eventually attributed to group II. Generally, the emission lines of group I occur more frequently than their group II counterparts. While almost all spectra in Fig. 6 show emission lines $\approx 5-10 \mathrm{meV}$ below $X_{A}$ (partially overlapping likely due to the high density of associated excitonic complexes), only a few apertures show a clear signature of the emission lines belonging to group II. Here, apertures $A 5-A 12$ illustrate the rare cases of these group II emission lines that we extracted from $\mu$-PL measurements performed on more than 50 apertures, which all showed emitters related to group I.

The classification of these sharp emission lines into the groups I and II becomes clearer based on Fig. 7, showing excitation-power-dependent $\mu$-PL spectra for aperture $A 3$. This excitation power series shows a sequence of spectra that is typical for all apertures showing emission lines of groups I and II. When varying the excitation power density by a factor of 10, group I emission lines exhibit only minor energetic shifts on the order of $\approx 100 \mu \mathrm{eV}$. In contrast, the emission line of group II from Fig. 7 shifts by $\approx 2 \mathrm{meV}$ toward lower energies.

The emission lines of group I seem to behave like common bound excitons in $\mathrm{GaN}$ upon rising excitation power that do not exhibit any pronounced energetic shifts in contrast to other semiconductors [62,104]. At the given low indium content of $0.37 \%$ (cf. Figs. 6 and 7) and the applied low excitation power density for the $\mu$-PL measurements (see Fig. 6), we do not expect any pronounced deviation from this common behavior of bulk GaN. Consequently, we assume that the emission lines of group I belong to $\mathrm{Si}^{0} X_{A}-\operatorname{In}^{n}$ assemblies, comprising a unique configuration of indium atoms within the Bohr radius of the exciton $r_{B}$. Hence, these group I emitters can be referred to as spatially direct, neutral, complex bound excitons.

It is important to note that the excitation power density applied for all $\mu$-PL measurements shown in Figs. 5-7 corresponds to low excitation conditions. For instance, the excitation power density is around 2 orders of magnitude lower than that commonly required to observe biexcitonic emission in, e.g., single GaN/AIN QDs excited below the wetting layer energy $[105,106]$. Furthermore, the maximum excitation power density applied in Fig. 7 yields carrier densities, which are still around 4-5 orders of magnitude below the Mott transition from an excitonic 
to a plasmalike regime in $\mathrm{GaN}[107,108]$. Hence, any optical signatures stemming from many-body effects induced by high excitation can safely be disregarded in our $\mu$-PL spectra. Because of the low excitation power density conditions, long integration times on the order of tens of minutes are required for recording $\mu$-PL spectra, which also necessitates an overall high setup stability.

\section{E. Impact of spectral diffusion for group II $\mathrm{Si}^{0} X_{A}-\mathbf{I n}^{n}$ based emitters}

The pronounced redshift of the emission lines belonging to group II points toward a gradual change in the effective Coulomb interaction between the charge particles attached to the $\mathrm{Si}^{0} X_{A}-\operatorname{In}^{n}$ assembly, which already occurs in the limit of low excitation powers. A spatially indirect transition for the group II emitters could explain this particular sensitivity to the excitation power density as well as the larger $E_{\text {loc }}$ values with respect to the emission lines of group I. Clearly, with rising $x$ such spatially indirect transitions involving $\mathrm{In}^{n}$ assemblies with increasing size and density become more probable. In a spatially indirect, neutral, complex bound exciton (group II), the exciton can be distributed over a particular $\operatorname{In}^{n}$ assembly and a single $\mathrm{Si}^{0}$ center over distances in excess of $r_{B}$. In this context we may assume that the hole is preferentially localized at an $\operatorname{In}^{n}$ assembly [28]. At first glance, the observation of group II emitters resembles the common picture of excitons trapped in $c$-plane (In)GaN QDs that are embedded in, e.g., an $\mathrm{Al}_{x} \mathrm{Ga}_{1-x} \mathrm{~N}(0 \leq x \leq 1)$ matrix [109]. Charge fluctuations typically occur in the matrix material due to point defects and surface charges [110], which in turn leads

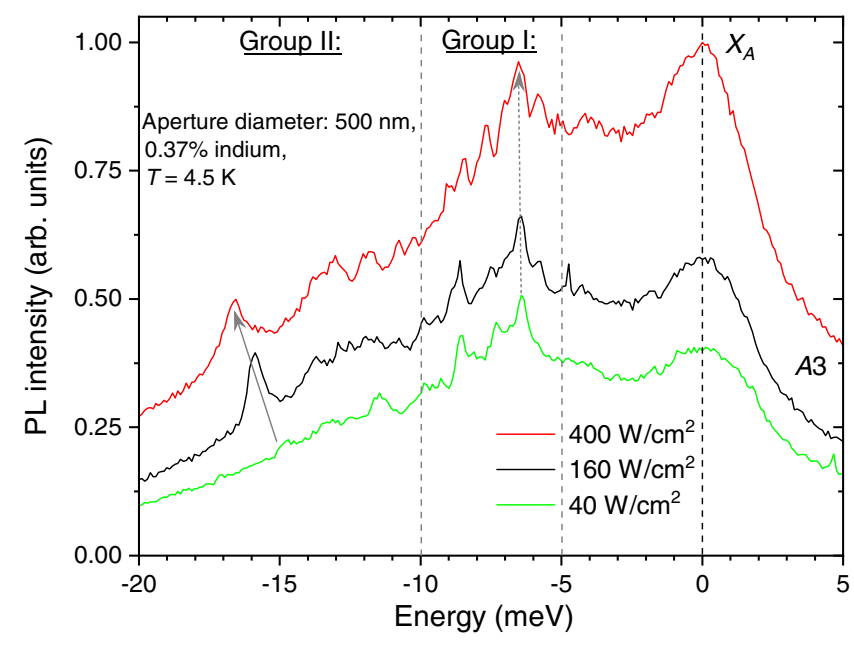

FIG. 7. Excitation-power-dependent $\mu$-PL measurements for the third aperture $(A 3)$ from Fig. 6(a). Emission lines of group I only exhibit shifts on the order of $100 \mu \mathrm{eV}$ upon a tenfold increase in excitation power density (see dashed gray arrow), while the distinct emission line of group II shifts by $\approx 2 \mathrm{meV}$ (see solid gray arrow). Similar observations hold for all apertures analyzed in Fig. 6. to a pronounced QD linewidth broadening known to be particularly strong in III-nitrides [111]. Upon changing the laser excitation power density, the occupation probability of the charge traps distributed in the vicinity of such QDs is altered [112]. As a result of this statistical process, a redshift of single nitride QD emission lines can be observed with rising excitation power density $[106,109,113]$, which resembles the emission line shift of the group II emitter exemplified in Fig. 7. Hence, the pronounced spectral shift of group II emission lines upon varying excitation power density could originate from the so-called spectral diffusion phenomenon, which scales with the size of the excitonic dipole moment [111]. We wish to note that this particular redshift of the QD emission lines does not originate from a screening of the quantum-confined Stark effect [114] because low excitation power densities are maintained. In addition, the screening of any built-in fields induced by pyro- and piezoelectricity should be negligible in our bulk InGaN samples with a thickness of $100 \mathrm{~nm}$.

Berthelot et al. first introduced a microscopic model of spectral diffusion in order to describe the excitation-powerdependent linewidth evolution they observed for individual InAs/GaAs QDs [112,115]. Later, Kindel [109] adapted this model to individual GaN/AlN QDs in order to describe an emission redshift upon increasing excitation power. The excitation power $(P)$ dependence for the emission energy of a single exciton emitter $\left(E_{X}\right)$ can be described by the following equation $[109,115]$ :

$$
E_{X}(P)=E_{X, 0}+d E_{\max } \frac{1}{\sqrt{P / P_{50 \%}}+1} .
$$

Herein, $E_{X, 0}$ describes the emission energy of the emitter for high $P$. As a result, all charges are removed from their trapping potential, which in turn also minimizes charge fluctuations at the origin of spectral diffusion. In addition, $d E_{\max }$ is the difference between $E_{X, 0}$ and $E_{X}(P \rightarrow 0)$, whereas $P_{50 \%}$ is the excitation power for which the occupation probability of the charge traps yields $50 \%$. Generally, Eq. (4) can describe a redshift as well as a blueshift, depending on the nature of the charges and their particular distribution around the excitonic emitter. Generally, an asymmetry in the charge distribution can be caused in our samples by surface charges, but also by the $\mathrm{AlGaN}$ layer beneath the 100 -nm-thick InGaN layer. Generally, the phenomenon of spectral diffusion is especially suited to account for the experimental observation in the low excitation power density regime shown in Fig. 7, because elevated pump power densities would otherwise already strongly quench the charge fluctuations occurring in the vicinity of group II emitters. As a consequence, no more redshift could be observed. Clearly, spatially indirect excitonic transitions of group II exhibit a larger excitonic dipole moment compared to their group I counterparts and are consequently more sensitive to the presence of any 
charge fluctuations. Future work could investigate whether any preferential orientation exists for excitonic dipoles related to group II emitters. Currently, a more detailed analysis of these emitters and a comparison of the experimental results and Eq. (4) is hindered by the spectral overlap between emitters, hence, preventing a detailed $E_{X}(P)$, linewidth, and emission line jitter analysis as commonly applied for III-nitride QDs [96,116].

\section{DISCUSSION}

More detailed $\mu$-PL measurements need to be performed based on specially designed samples in order to reach a conclusive picture regarding the sharp emission lines shown in Figs. 5-7. The spectra for apertures $A 1$ and $A 2$ shown in Fig. 6(a) include a first hint for the preferential formation of certain $\mathrm{Si}^{0} X_{A}-\mathrm{In}^{n}$ assemblies. Even though both spectra were recorded at completely different sample positions (several tens of micrometers apart), the overall optical signature is strikingly similar. In addition, future temperature-dependent $\mu$-PL measurements should provide further insight into the underlying physics. So far, we only observed that the emission lines displayed in Fig. 6 rapidly broaden and thermalize (not shown, traceable up to $\sim 30 \mathrm{~K}$ ) due to the associated exciton-phonon coupling involving $E_{2}^{\text {low }}$ phonons, localized phonon modes, and acoustic phonons, as described in Figs. 4 and 5. The distinction made between emission lines of group I and II is mainly motivated by the experiment and helps to access and to a certain degree also to identify the mechanisms of alloying. Generally, a continuous transition can be expected in between these two groups of emission lines, because with rising $x$ the probability for the formation of spatially indirect transitions should first increase in the dilute alloy limit.

The appearance of spatially direct and indirect $\mathrm{Si}^{0} X_{A}-$ $\operatorname{In}^{n}$ recombinations is likely interlinked with the $x$ dependence of the homogeneous linewidth broadening $\Gamma(T, x)$ shown in Fig. 4(a). Spatially more indirect transitions exhibit larger excitonic dipole moments that would enable a strong coupling to, e.g., polar phonons like LO phonons via the Fröhlich interaction [118]. However, the limited $E_{\text {loc }}$ values of excitonic $\mathrm{Si}^{0} X_{A}-\operatorname{In}^{n}$ complexes render the contribution of the exciton-LO-phonon coupling negligible in the present temperature range (due to the large LO-phonon energies in III-nitrides $[88,89])$ and the coupling to acoustic, localized, and nonpolar, e.g., $E_{2}^{\text {low }}$, phonons becomes more relevant, as introduced in the context of Fig. 4. The associated exciton-phonon interaction is dominated by the deformation potential coupling, which does not directly scale with the excitonic dipole moment. In addition, a less prominent piezoelectric coupling will occur [118]. Hence, we can expect the emission of excitonic $\mathrm{Si}^{0} X_{A}-\mathrm{In}^{n}$ complexes with large $E_{\text {loc }}$ values (group II) to be more temperature stable [119] than their group I counterparts as long as a dominant contribution of LO phonons can be excluded.
Therefore, with rising temperature the overall emission band related to $\mathrm{Si}^{0} X_{A}-\mathrm{In}^{n}$ assemblies should first become increasingly influenced by assemblies with effectively larger $E_{\text {loc }}$ values (group II), while the more spatially direct recombination channels (group I) should preferentially dissociate. However, already at $12 \mathrm{~K}$ the emitters of group II seem to increasingly contribute to the linewidth broadening of $\mathrm{Si}^{0} X_{A}$ with rising $x$. Thus, this could partially explain the offset observed between the associated experimental $\Delta E$ values and the model (solid red line) that increases with $x$ as shown in Fig. 3(a).

Finally, we suggest the following picture for the particular evolution of $\Gamma(T, x)$. At the onset of alloy formation one can observe a local indium enrichment in the vicinity of $\mathrm{Si}^{0}$ centers that affects the exciton-phonon coupling. As a result, more and more localized phonons contribute to the exciton-phonon coupling that clearly governs the observed $\Gamma(T, x)$ trend. However, in addition, spatially direct and indirect $\mathrm{Si}^{0} X_{A}-\mathrm{In}^{n}$ transitions should exhibit different thermalization behaviors, which possibly contributes to the particular evolution of $\Gamma(T, x)$. Already in the PL spectrum of the sample with $x=2.4 \%$ in Fig. 2, one can find a first hint for such an asymmetric emission line broadening caused by complexes with larger $E_{\mathrm{loc}}$ values. The low-energy tail of the $\mathrm{Si}^{0} X_{A}$ emission deviates from the displayed simple fitting procedure as, e.g., an increasing number of larger indium-related assemblies already contributes to the tail emission. However, a detailed line shape modeling as well as a quantification of the respective weighting between the different effects that lead to the particular evolution of $\Gamma(T, x)$ are beyond the scope of the present work and represent a motivating task for future studies.

The observed preferential incorporation of indium atoms next to $\mathrm{Si}^{0}$ impurities at the onset of alloy formation also reflects a deviation from the common random alloy conception, which formed the basis for the modeling illustrated in Fig. 3(a). With rising indium content $x$, this deviation from randomness should become increasingly masked from the perspective of the $\mathrm{Si}^{0} X_{A}$ excitonic complex. In contrast, the $X_{A}$ complex should always monitor a random alloy within the experimental constraints of the present study, cf. Sec. V. Consequently, the degree of randomness observed for the distribution of indium atoms depends on the nature of the excitonic complex, which is employed as a probe to monitor the alloy. Generally, a more localized alloy probe like $\mathrm{Si}^{0} X_{A}$ will require a higher indium content in order to monitor the entire material as a truly random alloy. Interestingly, even such a localized alloy probe will monitor a fraction of the entire alloy as a random alloy within the boundaries of the corresponding averaging volume.

\section{OUTLOOK}

Interestingly, the present work reveals an entire set of different excitonic complexes suitable for a nanoscopic 
study of a class II alloy. Hence, not only the advanced techniques listed in Sec. I, such as APT, STEM, nanoSIMS, and CL, are capable of analyzing a mixed-crystal alloy at the nanoscale. Even standard PL and low excitation $\mu$-PL measurements allow probing dimensions at least down to the order of $r_{B}$ in bulk semiconductor samples. Here, optical spectroscopy is not only limited to the direct emission fingerprint of different excitons (see, for instance, Figs. 2 and 6), but also the exciton-phonon coupling proves to be a powerful tool for studying alloy formation [see Figs. 4 and 5(b)]. Especially the latter point is promising for future class II alloy studies, as the exciton-phonon coupling was already successfully utilized to distinguish donor- or acceptorlike isoelectronic centers in class I alloys as summarized in the pioneering work of Hopfield et al. [31]. Hence, clearly future studies should focus on the phonon replicas related to the $\mathrm{Si}^{0} X_{A}-\mathrm{In}^{n}, \mathrm{Si}^{0} X_{A}$, and $X_{A}$ centers identified and utilized in the present work. As a result, the determination of all related exciton-phonon coupling constants would be of high relevance, e.g., for device-related modeling approaches [120].

A more detailed spectroscopic analysis of the $X_{A}$ complex at the onset of alloy formation in $\mathrm{InGaN}$ or other mixed-crystal alloys would directly be related to a reduction of the probe volume below the limits of standard $\mu$-PL measurements (this work). Recently, Hahn et al. [121] reported on luminescence spectra of InGaN/GaN QWs, which were obtained by injecting electrons from a scanning tunneling microscope (STM) tip. This represents a very promising approach as the spatial resolution can reach the few-nanometer range. Performing similar measurements at cryogenic temperatures would likely provide useful insights into the spatial distribution and substructure of different $\mathrm{Si}^{0} X_{A}-\mathrm{In}^{n}$ and $X_{A}-\operatorname{In}^{n}$ assemblies. Even more interestingly, based on such a high spatial resolution technique, the entire general spectroscopic approach from the present work could be transferred to InGaN samples with higher, and hence more technologically relevant, indium contents due to the smaller probe volume, cf. Fig. 5(a). Naturally, this also represents a promising pathway for a nanoscopic study of other semiconductor alloy systems.

The strong impact of alloying on the exciton-phonon coupling related to the $\mathrm{Si}^{0} X_{A}-\operatorname{In}^{n}$ assemblies introduced in Fig. 4 could also be investigated by resonant and nonresonant Raman measurements. In particular, localized phonon modes could directly be probed by $\mu$-Raman or even tip-enhanced Raman spectroscopy (TERS). In this latter case, note that a spatial resolution below $35 \mathrm{~nm}$ was achieved by Poliani et al. [122] on InGaN/GaN multiple QW structures embedded in GaN nanorods. However, in general, a spatial resolution comparable to the aforementioned STM technique should be reachable by TERS [123], which would enable a direct analysis of the localized phonon modes hypothesized in Secs. III B and III C.

\section{CONCLUSIONS}

In summary, we have demonstrated a detailed spectroscopic analysis of a III-V mixed-crystal alloy by PL and $\mu$-PL leading to a macroscopic and even a microscopic material characterization. Thus, the present work on a class II alloy approaches the high level of spectroscopic sophistication previously known only for class I alloys that relies on the emission of excitons bound to isoelectronic centers. As no such strongly localized excitons appear in the investigated $\operatorname{In}_{x} \mathrm{Ga}_{1-x} \mathrm{~N}$ epilayers $(0 \leq x \leq 2.4 \%)$, we utilized shallow impurities forming bound states as a probe to study the particular distribution of isoelectronic centers at the onset of alloy formation. By means of $\mu$-PL we directly observed a hierarchy of bound excitons related to dilute silicon-indium assemblies as individual, energetically sharp $(\mathrm{FWHM} \approx 300 \mu \mathrm{eV}$ ) emission lines appear. Consequently, we introduced a classification of the underlying emitters into spatially direct and indirect bound excitonic complexes, whose balance is weighted by the indium-induced localization of charge carriers at the very onset of alloy formation. Here, the spatially indirect bound excitonic complexes appeared as particularly sensitive to the phenomenon of spectral diffusion. However, not only such $\mu$-PL data but even conventional macro-PL spectra allowed us to extract crucial material parameters for the mixedcrystal alloy at hand. Based on ensembles of impurity bound excitons (three-particle complexes), we studied the indium-enriched environment of neutral silicon donors in InGaN at the length scale of the exciton Bohr radius. The analysis of the related exciton-phonon coupling revealed a reduction of the effective optical phonon energy from $12.9 \pm 0.4$ to $6.4 \pm 1.0 \mathrm{meV}$ upon rising indium content $(0 \leq x \leq 1.36 \%)$. This particular trend was explained by the phenomenon of phonon localization induced by a local enrichment of the immediate $\mathrm{Si}^{0}$ environment with isoelectronic centers. Interestingly, for the related $\mathrm{Si}^{0} X_{A}$ complex the alloying dependence of the homogeneous emission line broadening is additionally influenced by an indium-induced delocalization of the exciton. Based on the luminescence traces of free excitons that become increasingly trapped upon alloy formation, we extracted microscopic material properties from conventional PL data. We showed that upon increasing indium content $x$ the radius of the excitonic averaging volume $r_{a}$ reduces gradually from $27.2 \pm 3.7 \mathrm{~nm}$ at $x=0.01 \%$ down to $5.7 \pm 1.3 \mathrm{~nm}$ at $x=2.4 \%$. This transition illustrates the evolution from a free exciton in a doped semiconductor to a bound exciton (two-particle complex) in a class II alloy. As a result, the exciton capture by point and structural defects will diminish, supporting the high $\eta_{\text {int }}$ values of the InGaN alloy even at defect densities that are otherwise detrimental for other III-V binary semiconductors and alloys like, e.g., (In)GaAs [36,124], AlInGaP [35], and AlGaAs [21,36]. Furthermore, single indium atoms do not introduce any electronic state in the band gap of InGaN as confirmed by our PL experiments 
and first theoretically predicted by Bellaiche et al. [28]. Clearly, this justifies the categorization of InGaN as a class II alloy. The present results open the perspective to utilize not only macro-PL spectra but also the emission of individual excitonic complexes as powerful tools for probing any class II alloy at the few-nanometer scale. In this regard, our results are not limited to the InGaN alloy. However, the constraints regarding a suitable doping interval are strict and explainto the best of our knowledge- the absence of corresponding data in the literature for any other class II alloys.

\section{ACKNOWLEDGMENTS}

This work is supported by the Marie Skłodowska-Curie action "PhotoHeatEffect" (Grant No. 749565) within the European Union's Horizon 2020 research and innovation program and the FNS Project No. 200021E 175652. The authors wish to thank I. M. Rousseau for his support with the experimental setup. Furthermore, we highly acknowledge D. Martin for growing the samples, K. Shojiki for processing the metal apertures, and J.-F. Carlin for performing high-resolution x-ray diffraction measurements, cf. Sec. I of SM [52].

[1] D. G. Thomas, J. J. Hopfield, and C. J. Frosch, Isoelectronic Traps due To Nitrogen in Gallium Phosphide, Phys. Rev. Lett. 15, 857 (1965).

[2] W. Czaja, Festkörperprobleme XI, in Advances in Solid State Physics, edited by O. Madelung (Pergamon, Vieweg, 1971), pp. 65-85.

[3] P. J. Dean, D. G. Thomas, and C. J. Frosch, New Isoelectronic Trap Luminescence in Gallium Phosphide, J. Phys. C 17, 747 (1984).

[4] Y. Zhang, B. Fluegel, A. Mascarenhas, H. P. Xin, and C. W. Tu, Optical Transitions in the Isoelectronically Doped Semiconductor GaP:N: An Evolution from Isolated Centers, Pairs, and Clusters to an Impurity Band, Phys. Rev. B 62, 4493 (2000).

[5] R. A. Faulkner and P. J. Dean, Electronic Structure of Ground and Excited States of Isoelectronic Traps, J. Lumin. 1, 2, 552 (1970).

[6] S. Francoeur, S. Tixier, E. Young, T. Tiedje, and A. Mascarenhas, Bi isoelectronic Impurities in GaAs, Phys. Rev. B 77, 085209 (2008).

[7] Z. Xue-shu and L. Guo-hua, Nitrogen Isoelectronic Traps in GaAs, Chin. Phys. Lett. 1, 19 (1984).

[8] D. J. Wolford, J. A. Bradley, K. Fry, and J. Thompson, in Proceedings of the 17th International Conference on the Physics of Semiconductors (Springer, New York, 1985), pp. 627-630.

[9] J. L. Merz, Isoelectronic Oxygen Trap in ZnTe, Phys. Rev. 176, 961 (1968).

[10] T. Fukushima and S. Shionoya, Two Types of Luminescence Transitions in CdS Involving Te Isoelectronic Traps, Jpn. J. Appl. Phys. 15, 813 (1976).
[11] J. Cullen, K. Johnston, D. Dunker, E. McGlynn, D. R. Yakovlev, M. Bayer, and M. O. Henry, The Hg Isoelectronic Defect in ZnO, J. Appl. Phys. 114, 193515 (2013).

[12] W. M. Jadwisienczak and H. J. Lozykowski, Luminescence Properties of As, $P$, and Bi as Isoelectronic Traps in GaN, Mater. Res. Soc. Symp. Proc. 482, 1033 (1997).

[13] T Mattila and A. Zunger, $P-P$ and As-As Isovalent Impurity Pairs in GaN: Interaction of Deep $t_{2}$ Levels, Phys. Rev. B 59, 9943 (1999).

[14] R. A. Faulkner, Toward a Theory of Isoelectronic Impurities in Semiconductors, Phys. Rev. 175, 991 (1968).

[15] J. C. Phillips, Cancelation Theorem for Isoelectronic Impurity Binding Energies, Phys. Rev. Lett. 22, 285 (1969).

[16] J. W. Allen, Isoelectronic Impurities in Semiconductors: A Survey of Binding Mechanisms, J. Phys. C 4, 1936 (1971).

[17] R. Braunstein, A. R. Moore, and F. Herman, Intrinsic Optical Absorption in Germanium-Silicon Alloys, Phys. Rev. 109, 695 (1958).

[18] J. J. Tietjen and L. R. Weisberg, Electron Mobility in $\mathrm{GaAs}_{1-x} \mathrm{P}_{x}$ Alloys, Appl. Phys. Lett. 7, 261 (1965).

[19] I. A. Lamkin, S. A. Tarasov, A. V. Solomonov, and I. I. Mikhailov, Influence of the Composition of the GaAsP Solid Solution on the Ag-GaAsP Barrier Height, J. Phys. Conf. Ser. 690, 012037 (2016).

[20] J. P. Laurenti, P. Roentgen, K. Wolter, K. Seibert, H. Kurz, and J. Camassel, Indium-Doped GaAs: A Very Dilute Alloy System, Phys. Rev. B 37, 4155 (1988).

[21] E. F. Schubert, E. O. Göbel, Y. Horikoshi, K. Ploog, and H. J. Queisser, Alloy Broadening in Photoluminescence Spectra of $\mathrm{Al}_{x} \mathrm{Ga}_{1-x} \mathrm{As}$, Phys. Rev. B 30, 813 (1984).

[22] R. Butté, L. Lahourcade, T. K. Uždavinys, G. Callsen, M. Mensi, M. Glauser, G. Rossbach, D. Martin, J.-F. Carlin, S. Marcinkevičius, and N. Grandjean, Optical Absorption Edge Broadening in Thick InGaN Layers: Random Alloy Atomic Disorder and Growth Mode Induced Fluctuations, Appl. Phys. Lett. 112, 032106 (2018).

[23] M. C. Wagener, G. R. James, A. W. R. Leitch, and F. Omnès, On the Nature of Si-Doping in AlGaN Alloys, Phys. Status Solidi C 1, 2322 (2004).

[24] G. R. James, A. W. R. Leitch, F. Omnès, M. C. Wagener, and M. Leroux, Correlation of Transport and Optical Properties of Si-Doped $\mathrm{Al}_{0.23} \mathrm{G}_{0.77} \mathrm{~N}$, J. Appl. Phys. 96, 1047 (2004).

[25] O. Goede, L. John, and D. Hennig, Compositional Disorder-Induced Broadening for Free Excitons in II-VI Semiconducting Mixed Crystals, Phys. Status Solidi B 89, K183 (1978).

[26] A. Klochikhin, A. Reznitsky, S. Permogorov, T. Breitkopf, M. Grün, M. Hetterich, C. Klingshirn, V. Lyssenko, W. Langbein, and J. M. Hvam, Luminescence Spectra and Kinetics of Disordered Solid Solutions, Phys. Rev. B 59, 12947 (1999).

[27] M. Grundmann and C. P. Dietrich, Lineshape Theory of Photoluminescence from Semiconductor Alloys, J. Appl. Phys. 106, 123521 (2009).

[28] L. Bellaiche, T. Mattila, L. W. Wang, S. H. Wei, and A. Zunger, Resonant Hole Localization and Anomalous Optical Bowing in InGaN Alloys, Appl. Phys. Lett. 74, 1842 (1999). 
[29] P. J. Dean, Recombination Processes Associated with Deep States in Gallium Phosphide, J. Lumin. 1-2, 398 (1970).

[30] H. Kanzaki and S. Sakuragi, Optical Absorption and Luminescence of Excitons in Silver Halides Containing Isoelectronic Impurities. Part I. AgBr:I-, J. Phys. Soc. Jpn. 27, 109 (1969).

[31] J. J. Hopfield, D. G. Thomas, and R. T. Lynch, Isoelectronic Donors and Acceptors, Phys. Rev. 17, 312 (1966).

[32] D. G. Thomas and J. J. Hopfield, Isoelectronic Traps due to Nitrogen in Gallium Phosphide, Phys. Rev. 150, 680 (1966).

[33] A. Muller, P. Bianucci, C. Piermarocchi, M. Fornari, I. C. Robin, R. André, and C. K. Shih, Time-Resolved Photoluminescence Spectroscopy of Individual Te Impurity Centers in ZnSe, Phys. Rev. B 73, 081306(R) (2006).

[34] M. J. Manfra, Molecular Beam Epitaxy of Ultra-HighQuality AlGaAs/GaAs Heterostructures: Enabling Physics in Low-Dimensional Electronic Systems, Annu. Rev. Condens. Matter Phys. 5, 347 (2014).

[35] G. B. Stringfellow and M. G. Craford, in High Brightness Light Emitting Diodes, Semiconductors and Semimetals, Vol. 48 (Academic Press Inc., San Diego, 1998).

[36] S. F. Chichibu, A. Uedono, T. Onuma, B. A. Haskell, A. Chakraborty, T. Koyama, P. T. Fini, S. Keller, S. P. DenBaars, J. S. Speck, U. K. Mishra, S. Nakamura, S. Yamaguchi, S. Kamiyama, H. Amano, I. Akasaki, J. Han, and T. Sota, Origin of Defect-Insensitive Emission Probability in In-Containing (Al,In,Ga)N Alloy Semiconductors, Nat. Mater. 5, 810 (2006).

[37] M. Kneissl, T. Kolbe, C. Chua, V. Kueller, N. Lobo, J. Stellmach, A. Knauer, H. Rodriguez, S. Einfeldt, Z. Yang, N. M. Johnson, and M. Weyers, Advances in Group III-Nitride-Based Deep UV Light-Emitting Diode Technology, Semicond. Sci. Technol. 26, 014036 (2011).

[38] B. Gil, Physics of Wurtzite Nitrides and Oxides-Passport to Devices (Springer, Heidelberg, 2014).

[39] S. Nakamura, T. Mukai, and M. Senoh, High-Power GaN $P-N$ Junction Blue-Light-Emitting Diodes, Jpn. J. Appl. Phys. 30, L1998 (1991).

[40] S. Nakamura, S. Pearton, and G. Fasol, The Blue Laser Diode (Springer, Berlin, 1997).

[41] U. K. Mishra, L. Shen, T. E. Kazior, and Y.-f. Wu GaN-Based RF Power Devices and Amplifiers, Proc. IEEE 96, 287 (2008).

[42] S. Rajan and D. Jena, Gallium Nitride Electronics, Semicond. Sci. Technol. 28, 070301 (2013).

[43] C. Wächter, A. Meyer, S. Metzner, M. Jetter, F. Bertram, J. Christen, and P. Michler, High Wavelength Tunability of InGaN Quantum Wells Grown on Semipolar GaN Pyramid Facets, Phys. Status Solidi B 248, 605 (2011).

[44] F. Nippert, S. Yu Karpov, G. Callsen, B. Galler, T. Kure, C. Nenstiel, M. R. Wagner, M. Straßburg, H. J. Lugauer, and A. Hoffmann, Temperature-Dependent Recombination Coefficients in InGaN Light-Emitting Diodes: Hole Localization, Auger Processes, and the Green Gap, Appl. Phys. Lett. 109, 161103 (2016).

[45] F. A. Ponce and D. P. Bour, Nitride-Based Semiconductors for Blue and Green Light-Emitting Devices, Nature (London) 386, 351 (1997).
[46] A. Hangleiter, F. Hitzel, C. Netzel, D. Fuhrmann, U. Rossow, G. Ade, and P. Hinze, Suppression of Nonradiative Recombination by V-Shaped Pits in GaInN/GaN Quantum Wells Produces a Large Increase in the Light Emission Efficiency, Phys. Rev. Lett. 95, 127402 (2005).

[47] Y. Narukawa, Y. Kawakami, M. Funato, S. Fujita, S. Fujita, and S. Nakamura, Role of Self-Formed InGaN Quantum Dots for Exciton Localization in the Purple Laser Diode Emitting at 420 nm, Appl. Phys. Lett. 70, 981 (1997).

[48] P. R. C. Kent and A. Zunger, Carrier Localization and the Origin of Luminescence in Cubic InGaN Alloys, Appl. Phys. Lett. 79, 1977 (2001).

[49] M. J. Galtrey, R. A. Oliver, M. J. Kappers, C. J. Humphreys, D. J. Stokes, P. H. Clifton, and A. Cerezo, ThreeDimensional Atom Probe Studies of an $\operatorname{In}_{x} \mathrm{Ga}_{1-x} \mathrm{~N} / \mathrm{GaN}$ Multiple Quantum Well Structure: Assessment of Possible Indium Clustering, Appl. Phys. Lett. 90, 061903 (2007).

[50] A. Cerezo, P. H. Clifton, M. J. Galtrey, C. J. Humphreys, T. F. Kelly, D. J. Larson, S. Lozano-Perez, E. A. Marquis, R. A. Oliver, G. Sha, K. Thompson, M. Zandbergen, and R. L. Alvis, Atom Probe Tomography Today, Mater. Today 10, 36 (2007).

[51] L. Rigutti, B. Bonef, J. Speck, F. Tang, and R. A. Oliver, Atom Probe Tomography of Nitride Semiconductors, Scr. Mater. 148, 75 (2018).

[52] See Supplemental Material at http://link.aps.org/ supplemental/10.1103/PhysRevX.9.031030 for a description of (I.) the experimental details, (II.) the temperature-dependent photoluminescence traces, (III.) the temperature-dependent linewidth broadening, (IV.) a discussion of exciton diffusion, and (V.) additional information about the microphotoluminescence spectroscopy.

[53] H. Mathieu, P. Lefebvre, and P. Christol, Simple Analytical Method for Calculating Exciton Binding Energies in Semiconductor Quantum Wells, Phys. Rev. B 46, 4092 (1992).

[54] M. A. S. Kalceff and M. R. Phillips, Cathodoluminescence Microcharacterization of the Defect Structure of Quartz, Phys. Rev. B 52, 3122 (1995).

[55] K. Tanimura and N. Itoh, The Hopping Motion of the SelfTrapped Exciton in $\mathrm{NaCl}$, J. Phys. Chem. Solids 42, 901 (1981).

[56] A. K. Viswanath, J. I. Lee, D. Kim, C. R. Lee, and J. Y. Leem, Exciton-Phonon Interactions, Exciton Binding Energy, and Their Importance in the Realization of RoomTemperature Semiconductor Lasers Based on GaN, Phys. Rev. B 58, 16333 (1998).

[57] T. Onuma, T. Shibata, K. Kosaka, K. Asai, S. Sumiya, M. Tanaka, T. Sota, A. Uedono, and S. F. Chichibu, Free and Bound Exciton Fine Structures in AlN Epilayers Grown by Low-Pressure Metalorganic Vapor Phase Epitaxy, J. Appl. Phys. 105, 023529 (2009).

[58] R. Zimmermann, Theory of Exciton Linewidth in II-VI Semiconductor Mixed Crystals, J. Cryst. Growth 101, 346 (1990).

[59] I. Vurgaftman and J. R. Meyer, Band Parameters for Nitrogen-Containing Semiconductors, J. Appl. Phys. 94, 3675 (2003). 
[60] B. Monemar, P. P. Paskov, J. P. Bergman, A. A. Toropov, T. V. Shubina, T. Malinauskas, and A. Usui, Recombination of Free and Bound Excitons in GaN, Phys. Status Solidi B 245, 1723 (2008).

[61] G. Callsen, T. Kure, M. R. Wagner, R. Butté, and N. Grandjean, Excited States of Neutral Donor Bound Excitons in GaN, J. Appl. Phys. 123, 215702 (2018).

[62] B. Šantic, C. Merz, U. Kaufmann, R. Niebuhr, H. Obloh, and K. Bachem, Ionized Donor Bound Excitons in GaN, Appl. Phys. Lett. 71, 1837 (1997).

[63] B. Monemar, W. M. Chen, P. P. Paskov, T. Paskova, G. Pozina, and J. P. Bergman, The $3.466 \mathrm{eV}$ Bound Exciton in GaN, Phys. Status Solidi B 228, 489 (2001).

[64] R. Stepniewski, A. Wysmołek, M. Potemski, K. Pakuła, J. M. Baranowski, I. Grzegory, S. Porowski, G. Martinez, and P. Wyder, Fine Structure of Effective Mass Acceptors in Gallium Nitride, Phys. Rev. Lett. 91, 226404 (2003).

[65] S. Fischer, D. Volm, D. Kovalev, B. Averboukh, A. Graber, H. C. Alt, and B. K. Meyer, Shallow Donors in Epitaxial GaN, Mater. Sci. Eng. B 43, 192 (1997).

[66] C. Wetzel, T. Takeuchi, S. Yamaguchi, H. Katoh, H. Amano, and I. Akasaki, Optical Band Gap in $\mathrm{Ga}_{1-x} \mathrm{In}_{x} \mathrm{~N}$ $(0<x<0.2)$ on GaN by Photoreflection Spectroscopy, Appl. Phys. Lett. 73, 1994 (1998).

[67] C. Klingshirn, Semiconductor Optics, 2nd ed. (Springer, Berlin, 2005).

[68] G. Neu, A. A. Mbaye, and R. Triboulet, Excitons Spectroscopy in CdZnTe Alloys: Homogeneous and Inhomogeneous Broadening, in Proceedings of the 17th International Conference on the Physics of Semiconductors, San Francisco, Californian, USA (Springer-Verlag, New York, 1985), p. 1029.

[69] B. Gil, P. Bigenwald, M. Leroux, P. P. Paskov, and B. Monemar, Internal Structure of the Neutral DonorBound Exciton Complex in Cubic Zinc-Blende and Wurtzite Semiconductors, Phys. Rev. B 75, 085204 (2007).

[70] J. A. Kash, Exciton Tunneling Inhibited by Disorder in $\mathrm{GaAs}_{1-x} \mathrm{P}_{x}: \mathrm{N}$, Phys. Rev. B 29, 7069(R) (1984).

[71] H. Mariette, J. A. Kash, D. J. Wolford, and A. Marbeuf, Exciton Transfer at Low Temperature in $\mathrm{Ga}_{x} \mathrm{In}_{1-x} \mathrm{P}: \mathrm{N}$ and $\mathrm{GaAs}_{1-x} \mathrm{P}_{x}: \mathrm{N}$, Phys. Rev. B 31, 5217 (1985).

[72] A. Müller and M. Grundmann, Tunneling Dynamics of Excitons in Random Semiconductor Alloys, Phys. Rev. B 87, 035134 (2013).

[73] E. F. Schubert and W. T. Tsang, Photoluminescence Line Shape of Excitons in Alloy Semiconductors, Phys. Rev. B 34, 2991(R) (1986).

[74] S. F. Chichibu, T. Onuma, M. Kubota, A. Uedono, T. Sota, A. Tsukazaki, A. Ohtomo, and M. Kawasaki, Improvements in Quantum Efficiency of Excitonic Emissions in ZnO Epilayers by the Elimination of Point Defects, J. Appl. Phys. 99, 093505 (2006).

[75] C. K. Shu, J. Ou, H. C. Lin, W. K. Chen, and M. C. Lee, Isoelectronic In-Doping Effect in GaN Films Grown by Metalorganic Chemical Vapor Deposition, Appl. Phys. Lett. 73, 641 (1998).

[76] X.-Q. Shen, P. Ramvall, P. Riblet, and Y. Aoyagi, Improvements of the Optical and Electrical Properties of GaN Films by Using In-Doping Method During Growth, Jpn. J. Appl. Phys. 38, L411 (1999).
[77] X.-Q. Shen and Y. Aoyagi, An Approach to Achieve Intense Photoluminescence of GaN, Jpn. J. Appl. Phys. 38, L14 (1999).

[78] H. Kumano, K.-i. Hoshi, S. Tanaka, I. Suemune, X.-Q. Shen, P. Riblet, P. Ramvall, and Y. Aoyagi, Effect of Indium Doping on the Transient Optical Properties of GaN Films, Appl. Phys. Lett. 75, 2879 (1999).

[79] W. Liu, J.-F. Carlin, N. Grandjean, B. Deveaud, and G. Jacopin, Exciton Dynamics at a Single Dislocation in GaN Probed by Picosecond Time-Resolved Cathodoluminescence, Appl. Phys. Lett. 109, 042101 (2016).

[80] Y. Hayama, I. Takahashi, and N. Usami, Controlling Impurity Distribution in Quasi-Mono Crystalline Si Ingot by Seed Manipulation for Artificially Controlled Defects Technique, Energy Procedia 124, 734 (2017).

[81] H. Morkoç, Handbook of Nitride Semiconductors and Devices, 1st ed. (Wiley, Weinheim, 2009), p. 537.

[82] Y. Toyozawa, Theory of Line-Shapes of the Exciton Absorption Bands, Prog. Theor. Phys. 20, 53 (1958).

[83] S. Rudin, T. L. Reinecke, and B. Segall, TemperatureDependent Exciton Linewidths in Semiconductors, Phys. Rev. B 42, 11218 (1990).

[84] J. Lee, E. S. Koteles, and M. O. Vassell, Luminescence Linewidths of Excitons in GaAs Quantum Wells below 150 K, Phys. Rev. B 33, 5512 (1986).

[85] A. J. Fischer, W. Shan, J. J. Song, Y. C. Chang, R. Horning, and B. Goldenberg, Temperature-Dependent Absorption Measurements of Excitons in GaN Epilayers, Appl. Phys. Lett. 71, 1981 (1997).

[86] R. J. Mendelsberg, M. W. Allen, S. M. Durbin, and R. J. Reeves, Photoluminescence and the Exciton-Phonon Coupling in Hydrothermally Grown $\mathrm{ZnO}$, Phys. Rev. B 83, 205202 (2011).

[87] R. P. Seisyan, V. A. Kosobukin, and M. S. Markosov, Excitons and Polaritons in AlGaAs Semiconductor Alloys, Semiconductors 40, 1287 (2006).

[88] G. Callsen, J. S. Reparaz, M. R. Wagner, R. Kirste, C. Nenstiel, A. Hoffmann, and M. R. Phillips, Phonon Deformation Potentials in Wurtzite GaN and ZnO Determined by Uniaxial Pressure Dependent Raman Measurements, Appl. Phys. Lett. 98, 061906 (2011).

[89] V. Y. Davydov, V. V. Emtsev, I. N. Goncharuk, A. N. Smirnov, V. D. Petrikov, V. V. Mamutin, V. A. Vekshin, S. V. Ivanov, M. B. Smirnov, and T. Inushima, Experimental and Theoretical Studies of Phonons in Hexagonal InN, Appl. Phys. Lett. 75, 3297 (1999).

[90] V. Y. Davydov, Y. E. Kitaev, I. N. Goncharuk, A. N. Smirnov, J. Graul, O. Semchinova, D. Uffmann, M. B. Smirnov, A. P. Mirgorodsky, and R. A. Evarestov, Phonon Dispersion and Raman Scattering in Hexagonal GaN and AlN, Phys. Rev. B 58, 12899 (1998).

[91] R. Pässler, Temperature Dependence of Fundamental Band Gaps in Group IV, III-V, and II-VI Materials via a Two-Oscillator Model, J. Appl. Phys. 89, 6235 (2001).

[92] D. Y. Song, M. Basavaraj, S. A. Nikishin, M. Holtz, V. Soukhoveev, A. Usikov, and V. Dmitriev, The Influence of Phonons on the Optical Properties of GaN, J. Appl. Phys. 100, 113504 (2006).

[93] E. O. Kane, Pollmann-Büttner Variational Method for Excitonic Polarons, Phys. Rev. B 18, 6849 (1978). 
[94] J. R. Haynes, Experimental Proof of the Existence of a New Electronic Complex in Silicon, Phys. Rev. Lett. 4, 361 (1960).

[95] G. Callsen, M. Wagner, T. Kure, J. S. Reparaz, M. Bügler, J. Brunnmeier, C. Nenstiel, A. Hoffmann, M. Hoffmann, J. Tweedie, Z. Bryan, S. Aygun, R. Kirste, R. Collazo, and Z. Sitar, Optical Signature of Mg-Doped GaN: Transfer Processes, Phys. Rev. B 86, 075207 (2012).

[96] G. Callsen, G. M. O. Pahn, S. Kalinowski, C. Kindel, J. Settke, J. Brunnmeier, C. Nenstiel, T. Kure, F. Nippert, A. Schliwa, A. Hoffmann, T. Markurt, T. Schulz, M. Albrecht, S. Kako, M. Arita, and Y. Arakawa, Analysis of the Exciton-LO-Phonon Coupling in Single Wurtzite GaN Quantum Dots, Phys. Rev. B 92, 235439 (2015).

[97] C. Nenstiel, M. Bügler, G. Callsen, F. Nippert, T. Kure, S. Fritze, A. Dadgar, H. Witte, J. Bläsing, A. Krost, and A. Hoffmann, Germanium-The Superior Dopant in n-Type GaN, Phys. Status Solidi RRL 9, 716 (2015).

[98] T. V. Bezyazychnaya, D. M. Kabanau, V. V. Kabanov, Y. V. Lebiadok, A. G. Ryabtsev, G. I. Ryabtsev, V. M. Zelenkovskii, and S. K. Mehta, Influence of Vacancies on Indium Atom Distribution in InGaAs and InGaN Compounds, Lith. J. Phys. 55, 10 (2015).

[99] Z. Liu, B. Fu, X. Yi, G. Yuan, J. Wang, J. Li, Luna, and I. Ferguson, Co-Doping of Magnesium with Indium in Nitrides: First Principle Calculation and Experiment, RSC Adv. 6, 5111 (2016).

[100] A. D. Rodrigues, M. P. F. De Godoy, C. Mietze, and D. J. As, Phonon Localization in Cubic GaN/AlN Superlattices, Solid State Commun. 186, 18 (2014).

[101] V. A. Fonoberov and A. A. Balandin, Interface and Confined Optical Phonons in Wurtzite Nanocrystals, Phys. Rev. B 70, 233205 (2004).

[102] L. Zhang, Uniform Description of Polar Optical Phonon States and Their Fröhlich Electron-Phonon Interaction Hamiltonians in Multi-Layer Wurtzite Nitride LowDimensional Quantum Structures, Superlattices Microstruct. 53, 113 (2013).

[103] K. Kornitzer, T. Ebner, K. Thonke, R. Sauer, C. Kirchner, V. Schwegler, M. Kamp, M. Leszczynski, I. Grzegory, and S. Porowski, Photoluminescence and Reflectance Spectroscopy of Excitonic Transitions in High-Quality Homoepitaxial GaN Films, Phys. Rev. B 60, 1471 (1999).

[104] D. C. Reynolds, D. C. Look, and B. Jogai, Combined Effects of Screening and Band Gap Renormalization on the Energy of Optical Transitions in $\mathrm{ZnO}$ and GaN, J. Appl. Phys. 88, 5760 (2000).

[105] G. Callsen, A. Carmele, G. Hönig, C. Kindel, J. Brunnmeier, M. R. Wagner, E. Stock, J. S. Reparaz, A. Schliwa, S. Reitzenstein, A. Knorr, A. Hoffmann, S. Kako, and Y. Arakawa, Steering Photon Statistics in Single Quantum Dots: From One- to Two-Photon Emission, Phys. Rev. B 87, 245314 (2013).

[106] G. Hönig, G. Callsen, A. Schliwa, S. Kalinowski, C. Kindel, S. Kako, Y. Arakawa, D. Bimberg, and A. Hoffmann, Manifestation of Unconventional Biexciton States in Quantum Dots, Nat. Commun. 5, 5721 (2014).

[107] F. Binet, J. Y. Duboz, J. Off, and F. Scholz, HighExcitation Photoluminescence in GaN: Hot-Carrier
Effects and the Mott Transition, Phys. Rev. B 60, 4715 (1999).

[108] C. Nenstiel, G. Callsen, F. Nippert, T. Kure, M. R. Wagner, S. Schlichting, N. Jankowski, M. P. Hoffmann, S. Fritze, A. Dadgar, A. Krost, A. Hoffmann, and F. Bechstedt, Electronic Excitations Stabilised by a Degenerate Electron Gas in Semiconductors, Commun. Phys. 1, 38 (2018).

[109] C. H. Kindel, Study on Optical Polarization in Hexagonal Gallium Nitride Quantum Dots, Ph. D. thesis, University of Tokyo, 2010.

[110] I. Rousseau, G. Callsen, G. Jacopin, J.-F. Carlin, R. Butté, and N. Grandjean, Optical Absorption and Oxygen Passivation of Surface States in III-Nitride Photonic Devices, J. Appl. Phys. 123, 113103 (2018).

[111] C. Kindel, G. Callsen, S. Kako, T. Kawano, H. Oishi, G. Hönig, A. Schliwa, A. Hoffmann, and Y. Arakawa, Spectral Diffusion in Nitride Quantum Dots: Emission Energy Dependent Linewidths Broadening via Giant Built-In Dipole Moments, Phys. Status Solidi RRL 8, 408 (2014).

[112] A. Berthelot, I. Favero, G. Cassabois, C. Voisin, C. Delalande, Ph. Roussignol, R. Ferreira, and J. M. Gérard, Unconventional Motional Narrowing in the Optical Spectrum of a Semiconductor Quantum Dot, Nat. Phys. 2, 759 (2006).

[113] M. Holmes, S. Kako, K. Choi, M. Arita, and Y. Arakawa, Spectral Diffusion and Its Influence on the Emission Linewidths of Site-Controlled GaN Nanowire Quantum Dots, Phys. Rev. B 92, 115447 (2015).

[114] S. Schlichting, G. M. O. Hönig, J. Müßener, P. Hille, T. Grieb, S. Westerkamp, J. Teubert, J. Schörmann, M. R. Wagner, A. Rosenauer, M. Eickhoff, A. Hoffmann, and G. Callsen, Suppression of the Quantum-Confined Stark Effect in Polar Nitride Heterostructures, Commun. Phys. 1, 48 (2018).

[115] A. Berthelot, Spectral Diffusion and Motional Narrowing in Quantum Dots, $\mathrm{Ph}$. D. thesis, Université Pierre et Marie Curie, 2008.

[116] G. Callsen and G. M. O. Pahn, Identifying Multi-Excitons in Quantum Dots: The Subtle Connection between Electric Dipole Moments and Emission Linewidths, Phys. Status Solidi RRL 9, 521 (2015).

[117] G. Hönig, S. Rodt, G. Callsen, I. A. Ostapenko, T. Kure, A. Schliwa, C. Kindel, D. Bimberg, A. Hoffmann, S. Kako, and Y. Arakawa, Identification of Electric Dipole Moments of Excitonic Complexes in Nitride-Based Quantum Dots, Phys. Rev. B 88, 045309 (2013).

[118] I. A. Ostapenko, G. Hönig, S. Rodt, A. Schliwa, A. Hoffmann, D. Bimberg, M. R. Dachner, M. Richter, A. Knorr, S. Kako, and Y. Arakawa, Exciton Acoustic-Phonon Coupling in Single GaN/AlN Quantum Dots, Phys. Rev. B 85, 081303(R) (2012).

[119] B. K. Meyer, J. Sann, S. Eisermann, S. Lautenschlaeger, M. R. Wagner, M. Kaiser, G. Callsen, J. S. Reparaz, and A. Hoffmann, Excited State Properties of Donor Bound Excitons in ZnO, Phys. Rev. B 82, 115207 (2010).

[120] A. David, C. A. Hurni, N. G. Young, and M. D. Craven, Field-Assisted Shockley-Read-Hall Recombinations in IIINitride Quantum Wells, Appl. Phys. Lett. 111, 233501 (2017). 
[121] W. Hahn, J. M. Lentali, P. Polovodov, N. Young, S. Nakamura, J. S. Speck, C. Weisbuch, M. Filoche, Y. R. Wu, M. Piccardo, F. Maroun, L. Martinelli, Y. Lassailly, and J. Peretti, Evidence of Nanoscale Anderson Localization Induced by Intrinsic Compositional Disorder in InGaN/GaN Quantum Wells by Scanning Tunneling Luminescence Spectroscopy, Phys. Rev. B 98, 045305 (2018).

[122] E. Poliani, M. R. Wagner, J. S. Reparaz, M. Mandl, M. Strassburg, X. Kong, A. Trampert, C. M. Sotomayor Torres, A. Hoffmann, and J. Maultzsch, Nanoscale Imaging of InN Segregation and Polymorphism in Single Vertically Aligned InGaN/GaN Multi Quantum Well
Nanorods by Tip-Enhanced Raman Scattering, Nano Lett. 13, 3205 (2013).

[123] B.-S. Yeo, J. Stadler, T. Schmid, R. Zenobi, and W. Zhang, Tip-Enhanced Raman Spectroscopy-Its Status, Challenges and Future Directions, Chem. Phys. Lett. 472, 1 (2009).

[124] K. Nozawa and Y. Horikoshi, Low Threading Dislocation Density GaAs on Si (100) with InGaAs/GaAs StrainedLayer Superlattice Grown by Migration-Enhanced Epitaxy, Jpn. J. Appl. Phys. 30, L668 (1991).

Correction: A project number was missing from the Acknowledgments section and has been inserted. 